\title{
Chemical evolution of the intra-cluster medium
}

\author{
A. Moretti ${ }^{1}$, L. Portinari ${ }^{2}$, and C. Chiosi ${ }^{1}$ \\ 1 Department of Astronomy, University of Padua, Vicolo dell'Osservatorio 2, 35122 Padova, Italy \\ e-mail: moretti, chiosi@pd.astro.it \\ 2 Theoretical Astrophysics Center, Juliane Maries Vej 30, 2100 Copenhagen Ø, Denmark \\ e-mail: lportina@tac.dk
}

Received 15 May 2002 / Accepted 18 June 2003

\begin{abstract}
The high metallicity of the intra-cluster medium (ICM) is generally interpreted on the basis of the galactic wind scenario for elliptical galaxies. In this framework, we develop a toy model to follow the chemical evolution of the ICM, formulated in analogy to chemical models for individual galaxies. The model computes the galaxy formation history (GFH) of cluster galaxies, connecting the final luminosity function (LF) to the corresponding metal enrichment history of the ICM. The observed LF can be reproduced with a smooth, Madau-plot like GFH peaking at $z \sim 1-2$, plus a "burst" of formation of dwarf galaxies at high redshift.

The model is used to test the response of the predicted metal content and abundance evolution of the ICM to varying input galactic models. The chemical enrichment is computed from "galactic yields" based on models of elliptical galaxies with a variable initial mass function (IMF), favouring the formation of massive stars at high redshift and/or in more massive galaxies. For a given final galactic luminosity, these model ellipticals eject into the ICM a larger quantity of gas and of metals than do standard models based on the Salpeter IMF.

However, a scenario in which the IMF varies with redshift as a consequence of the effect of the cosmic background temperature on the Jeans mass scale appears to be too mild to account for the observed metal production in clusters. The high iron-massto-luminosity-ratio of the ICM can be reproduced only by assuming a more dramatic variation of the typical stellar mass, in line with other recent findings. The mass in the wind-ejected gas is predicted to exceed the mass in galaxies by a factor of 1.5-2 and to constitute roughly half of the intra-cluster gas.
\end{abstract}

Key words. galaxies: clusters: general - galaxies: abundances - galaxies: intergalactic medium

\section{Introduction}

The popular galactic wind (GW) scenario for elliptical galaxies, introduced by Larson (1974) to account for their photometric properties, predicted as a side effect the pollution of the intra-cluster medium (ICM) with the chemical elements produced and expelled by individual galaxies (Larson \& Dinerstein 1975). Metals in the hot ICM were in fact detected soon afterwards (Mitchell et al. 1976; Serlemitsos et al. 1977).

Iron is generally used as tracer of the overall metallicity, being the best measured element in the hot ICM. Typical iron abundances in the ICM are around 0.2-0.3 solar (Renzini 1997; Fukazawa et al. 1998; Mushotzky 1999; Finoguenov et al. 2000, 2001). The ICM seems to be also rich in $\alpha$-elements, for which ASCA provided more firm estimates; data by Mushotzky et al. (1996) yield $\left[\frac{\alpha}{\mathrm{Fe}}\right] \simeq 0.2 \pm 0.3$ or, in the case of oxygen, $\left[\frac{\mathrm{O}}{\mathrm{Fe}}\right] \simeq 0.48_{-0.55}^{+0.24}$, which means, considering the uncertainty, a marginal over-abundance of oxygen relative to iron. Mushotzky et al. (1996) derive $\left[\frac{\mathrm{Si}}{\mathrm{Fe}}\right] \simeq 0.37_{-0.35}^{+0.17}$, in agreement with the analysis by Fukazawa et al. (1998), who further

Send offprint requests to: A. Moretti, e-mail: moretti@pd.astro.it suggest that $\left[\frac{\mathrm{Si}}{\mathrm{Fe}}\right]$ may increase with cluster richness/temperature. Ishimaru \& Arimoto (1997) and Arimoto et al. (1997) argue that considering the uncertainties and the revised value of the solar iron abundance, abundance ratios are consistent with solar. The first XMM and Chandra studies seem to indicate the same (Buote 2003).

Recent studies have revealed a more complex distribution of metals in the ICM. Gradients in the iron abundance have been detected with ASCA data (Finoguenov et al. 2000, 2001; White 2000), and confirmed by BeppoSax data (Irwin \& Bregman 2001; De Grandi \& Molendi 2001); in particular, sharp metallicity peaks in the central region seem to be typical of cooling flows/cD clusters (Fukazawa et al. 2000; Irwin \& Bregman 2001; De Grandi \& Molendi 2001). [ $\alpha / \mathrm{Fe}]$ ratios also seem to display a composite behaviour, increasing with cluster radius from $\sim$ solar in the central regions to supersolar, typical type II supernova $(\mathrm{SN})$, values in the outer regions (Finoguenov et al. 2000). The existence of gradients of metallicity and of abundance ratios is being confirmed by the first XMM and Chandra results on a few clusters (Kaastra et al. 2001; Tamura et al. 2001; Smith et al. 2002; Johnstone et al. 2002; Ettori et al. 2002), but no extensive cluster samples have yet been 
analyzed with these satellites (Buote 2003). It has been suggested recently that, in the presence of abundance gradients, emissivity-weighted estimates of the average metallicity might be higher than the true mass-weighted average, up to a factor of two (Pellegrini \& Ciotti 2001). We shall not discuss abundance gradients in this paper however, but only the global average chemical evolution of the ICM.

The source of such a large amount of metals in the ICM could be galactic, as in the original prediction by Larson, or reside in Population III pre-galactic objects (White \& Rees 1978; Loewenstein 2001). The distinct correlation between the iron mass in the ICM and the luminosity of elliptical and S0 galaxies,

$M_{\mathrm{ICM}}^{\mathrm{Fe}} \propto L_{V}^{\mathrm{E}+\mathrm{S} 0}$

shown by Arnaud et al. (1992), seems to favour galaxies as the sites of production of the metals in the ICM.

Accepting that the metals in the ICM originated in the E and S0 galaxies of the cluster, two mechanisms exist to extract the newly synthesized elements from the individual galaxies: the above mentioned GW and ram pressure stripping. Arguments have been given by Renzini (1997) favouring the GW scenario, mostly based on the observation that the "iron mass-to-luminosity ratio" is roughly constant independently of cluster richness and temperature, while the ram pressure mechanism should be more efficient, extracting more metals for a given stellar content, in richer clusters.

Though the role of ram pressure stripping is still debated (Mori \& Burkert 2000), from here on we will limit to the GW scenario for the pollution of the ICM, bearing in mind that the addition of other mechanisms (metal production in pregalactic objects and/or ram pressure stripping) would allow to inject even more metals into the ICM, further favouring the enrichment. In our models, GWs are powered by SN feedback (Chiosi 2000b), as typically assumed in literature although AGNs have been suggested as an alternative source of energy input (Renzini et al. 1993; Romano et al. 2002).

Over the past two decades, modelling the metallicity of the ICM has been the subject of a great deal of studies that we summarize briefly in the next section. In this paper we present a model for the chemical evolution of a cluster, in which galaxies eject part of their gas content by the GW mechanism, thus playing the role of stars in the classical models for the chemical evolution of the interstellar medium (Tinsley 1980; Matteucci 1997).

The plan of the paper is as follows. Section 2 shortly reviews the problem of the gas and metal content of the ICM, and previous studies in literature. In Sect. 3 we suggest that a non-standard, variable initial mass function (IMF) for the stellar content of a galaxy could improve upon our understanding of the problem, and discuss the corresponding galactic models. Section 4 gives some simple estimates of the global properties of clusters, as expected from galactic models with the variable IMF or with the standard Salpeter IMF; we introduce the concept of intra-cluster mass-to-light ratio (ICMLR) as a measure of the amount of ICM gas. In Sect. 5 we introduce our new model for the chemical evolution of a cluster as a whole, the underlying analogy between the inter-stellar and intra-cluster medium, the concepts of galactic formation rate (GFR) and of galactic initial mass function (GIMF), and the model equations. Section 6 contains the detailed discussion of a fiducial model, calibrated on the observed ICMLR and the present-day luminosity function (LF) of galaxies as the key constraint for the GFR and GIMF; a comparison between results with the Salpeter IMF and with the non-standard IMF is also made. In Sect. 7, a set of models is presented with different galaxy formation histories. In Sect. 8 we present cluster models computed on the base of galactic models especially selected to reproduce the correct metal content of clusters. In Sect. 9 we discuss the predicted $[\alpha / \mathrm{Fe}]$ abundance ratios in the ICM. Summary and conclusions are drawn in Sect. 10.

\section{Gas and metal in the ICM: Previous studies}

Various early studies investigated whether "standard" chemical models for galaxies can explain the amount of metals detected in the ICM (Vigroux 1977; Hinnes \& Biermann 1980; De Young 1978); by "standard" we mean a chemical model with the same physical ingredients (mainly, stellar IMF and yields) suited to reproduce the Solar Neighborhood. Amidst these early studies, we mention in particular the one by Matteucci \& Vettolani (1988) as the first attempt to link directly the metallicity of the ICM with the properties of the corresponding galaxy population. To this aim, the authors developed a modelling technique that has been widely adopted afterwards. Basing on a grid of models of elliptical galaxies with GW, they assigned to any given galaxy of final stellar mass $M_{*}$, or equivalently of present-day luminosity $L_{*}$, the corresponding masses of gas and iron ejected in the $\mathrm{GW}, M_{\mathrm{gas}}^{\mathrm{ej}}$ and $M_{\mathrm{Fe}}^{\mathrm{ej}}$. Integrating these quantities over the observed LF, they calculated the total masses of gas and iron globally ejected by the galactic population in the cluster. Their main conclusions were:

(1) the iron content of the ICM can be reproduced with a standard Salpeter IMF in the individual galaxies;

(2) the global amount of gas ejected as GW is much smaller than the observed mass of the ICM, hence the ICM must be mostly primordial gas which was never involved in galaxy formation.

Later on, this early success in reproducing $M_{\mathrm{Fe}}^{\mathrm{ICM}}$ turned out to be favoured by the low gravitational potential wells of model galaxies, calculated only on the basis of their luminous, baryonic component. Once the potential well of the dark matter halo is taken into account, the ejecta of SN Ia hardly escape the galaxy and the metal pollution of the ICM by GWs is much reduced (David et al. 1991b; Matteucci \& Gibson 1995). In this case, standard chemical models fail to reproduce the metal content of the ICM. Some non-standard scenarios were thus invoked to solve the riddle, such as:

- a more top-heavy IMF than the Salpeter one, with logarithmic slope $x \sim 1.0$ rather than the standard value $x=1.35$ (David et al. 1991a; Matteucci \& Gibson 1995; Gibson \& Matteucci 1997b,a; Loewenstein \& Mushotzky 1996);

- a bimodal IMF with an early generation of massive stars heavily polluting the ICM, followed by a more normal star 
formation phase producing the stars we see today (Arnaud et al. 1992; Elbaz et al. 1995).

These models, where SN II from massive stars play the main role in the metal pollution of the ICM, were further supported by the enhanced abundances of $\alpha$-elements with respect to iron detected with ASCA (Mushotzky et al. 1996).

Just as in the early work by Matteucci \& Vettolani (1988), most authors conclude that GWs cannot account for the huge amount of gas present in the ICM, which is $2-5$ times the mass in galaxies (Arnaud et al. 1992). The ICM must then consist, 50 to $90 \%$, of primordial gas.

Trentham (1994), on the base of the steep slope of the LF at the low luminosity end observed in clusters, suggested that all the intra-cluster gas could have originated in dwarf galaxies, since these are numerous in clusters and they are expected to eject a large fraction of their initial mass as GW, due to their shallow potential wells. This suggestion was discarded by Nath \& Chiba (1995) and by Gibson \& Matteucci (1997b), who calculated detailed models of dwarf galaxies and related GW ejection to show that galaxies cannot be the only source for the whole intra-cluster gas, even in the case of the steepest observed LF (hence the largest contribution from dwarfs).

The models were further refined by Martinelli et al. (2000) who made use of one-zone and multi-zone GW models of elliptical galaxies and studied the dependence of the ICM abundances with redshift. While the abundance ratios $[\mathrm{O} / \mathrm{Fe}]$ are in both cases within the observational uncertainties, the abundances $[\mathrm{Fe} / \mathrm{H}]$ are very large and require large dilutions by primordial gas.

Very recently, Pipino et al. (2002) showed that the Salpeter IMF might reproduce the observed metallicity of the ICM, provided $100 \%$ efficiency of energy feed-back is adopted for SN Ia. In this case, all the iron produced at late times escapes into the ICM in a continuous wind/outflow. The problem with this scenario, however, is that it inevitably predicts strongly sub-solar $[\alpha / \mathrm{Fe}]$ ratios in the ICM.

Finally, a recent attempt aimed at reproducing simultaneously the iron abundance, the ratio $[\mathrm{O} / \mathrm{Fe}]$, and the gas mass, was by Chiosi (2000b) who made use of multi-zone models of elliptical galaxies and adopted a non-standard IMF for their stellar content. More details on the models by Chiosi (2000b) will be discussed in the next section as our present model stems from that work.

\section{A non-standard IMF}

As reviewed in the previous section, a wealth of work in literature suggests that some non-standard scenario (or IMF) must be invoked to account for the metals in the ICM. We recall that a non-standard IMF has been suggested for elliptical galaxies also on the base of other, independent arguments:

- a top-heavy IMF $(x \sim 1.0)$ is better suited to reproduce the photometric properties of ellipticals (Arimoto \& Yoshii 1987);

- systematic variations of the IMF in ellipticals of increasing mass might explain the increase of the mass-to-light $(M / L)$ ratio with galactic luminosity, that is the so-called "tilt of the Fundamental Plane" (Larson 1986; Renzini \& Ciotti 1993; Zepf \& Silk 1996; Chiosi et al. 1998).

What physical reason may lead to a different IMF in different situations? From the theoretical point of view, a turnover of the IMF at low masses is expected, related to the Jeans scale (thermal support) and to the scale of magnetic support against gravitational collapse (Larson 1998; Padoan \& Nordlund 2002). The conditions of the ambient gas may thus influence the cut-off of the IMF at low masses, and hence the mass fraction of a stellar generation that is locked into ever-living, very low mass and low luminosity stars. A variation of this "locked-up fraction" is crucial for the efficiency of the metal enrichment produced by a stellar population (Tinsley 1980).

Larson (1998) suggested the following functional form of the IMF:

$$
\frac{\mathrm{d} N}{\mathrm{~d} \log M} \propto M^{-x} \exp \left(-\frac{M_{\mathrm{s}}}{M}\right)
$$

where $M_{\mathrm{s}}$ is a typical mass scale related to the Jeans mass. In brief, this IMF is a Salpeter power law down to a typical peak mass

$M_{\mathrm{p}} \sim \frac{M_{\mathrm{s}}}{x}$

below which there is an exponential cut-off. The peak mass varies with the temperature and density of the parent gas as expected from Jeans' law:

$M_{\mathrm{p}} \propto T^{2} \rho^{-\frac{1}{2}}$

In warmer and/or less dense gas, therefore, the typical peak mass $M_{\mathrm{p}}$ increases and the locked-up fraction is lower. Larson (1998), as well as Chiosi et al. (1998); Chiosi (2000b), pointed out that a minimum temperature for the star forming clouds is set by the cosmic microwave background, whose temperature increases with redshift, becoming higher than the typical temperature of present-day molecular clouds at $z>2$. At increasing redshift, the sole background then produces an increasing Jeans mass and hence an IMF skewed toward massive stars. Other heating sources, like feed-back from massive stars or the UV background, and a reduced cooling rate at low metallicities, may enhance the effect further.

From the observational point of view, the issue of the variation of the IMF with ambient conditions is still open and very much debated. In local studies, some authors underline that, within the uncertainties, data are consistent with a constant IMF (Kroupa 2002), others find evidence of variation, e.g. between cluster and field (Massey 1998) in clouds of different density (Briceño et al. 2002; Luhman et al. 2003), or between the disk and the halo of the Milky Way (Chabrier 2003).

The IMF of the early star formation activity at high redshift is even harder to probe. Hernandez \& Ferrara (2001) find indirect evidence in the local halo for a typical stellar mass scale (Larson-like) increasing with redshift. A similar scenario has been very recently advocated by Finoguenov et al. (2003) on the base of the different metal content in groups and clusters 
- and the different typical redshift of formation of their stellar content. Indications of a top-heavy IMF at high redshift $(z>$ 3-6) has been also found for Lyman Break Galaxies (Ferguson et al. 2002). All of this agrees with a number of recent theoretical studies suggesting that the first generations of stars were strongly skewed toward massive stars (Abel et al. 2002; Bromm et al. 2002; Chiosi 2000a, and references therein).

In view of these results and theoretical arguments it is certainly a legitimate working hypothesis to consider an IMF with a physical dependence on the environment, through the typical Jeans mass.

\subsection{Galactic models with the PNJ IMF}

A behaviour resembling the one described above is predicted by the theoretical IMF by Padoan et al. (1997, hereinafter PNJ), which features a peak mass

$M_{\mathrm{p}}=0.2 M_{\odot}\left(\frac{T}{10 \mathrm{~K}}\right)^{2}\left(\frac{n}{1000 \mathrm{~cm}^{-3}}\right)^{-\frac{1}{2}}\left(\frac{\sigma}{2.5 \mathrm{~km} \mathrm{~s}^{-1}}\right)^{-1}$

where $T, n$, and $\sigma$ are the gas temperature, number density, and velocity dispersion, respectively. Although the physical derivation of the PNJ IMF has been sometimes questioned (Scalo et al. 1998), one can still adopt it in galactic models as a tentative recipe yielding the typical behaviour expected for the Jeans mass; see Chiosi (2000b) for further discussion.

Chiosi et al. (1998) developed chemo-thermodynamical models following the thermodynamical evolution of the gas in an elliptical galaxy, and the corresponding variations in the IMF according to the PNJ recipe. The characteristics and behaviour of these models as a function of galactic mass and redshift of formation are discussed in full details in Chiosi et al. (1998) and Chiosi (2000b). Here, we briefly underline the qualitative trends with respect to (a) mass and (b) redshift of formation.

(a) At increasing galactic mass, the average density of the object $\rho$ decreases and the typical peak mass $M_{\mathrm{p}}$ increases, yielding a lower locked-up fraction.

(b) At increasing redshift of formation $z_{\text {for }}$, the temperature of the protogalactic gas increases, since it can never fall below the corresponding temperature of the cosmic microwave background, $T \geq T_{\mathrm{CMB}}\left(z_{\text {for }}\right)$, which increases with redshift; hence, the peak mass $M_{\mathrm{p}}$ is higher and the locked-up fraction is lower.

We remark here that the above mentioned trends are by no means drastic: the peak mass $M_{\mathrm{p}}$ hardly exceeds $1 M_{\odot}$, and the "high $M_{\mathrm{p}}$ " phase is limited to the early galactic ages; after the initial stage, in fact, the system reaches a sort of thermodynamical balance, with the peak mass and the IMF settling on quite standard values. The overall picture loosely resembles the bimodal behaviour suggested by Elbaz et al. (1995), with an early phase dominated by massive stars followed by a more normal star formation phase producing the low-mass stars we see today. However, in our models the IMF naturally and smoothly varies in time following a physical prescription, rather than an imposed bimodal behaviour.
Though not long-lasting, the variations in the early phases suffice to differentiate the resulting galactic models, making them successful at reproducing many features of observed ellipticals, such as (Chiosi et al. 1998):

- the tilt of the Fundamental Plane;

- the analogous of the "G-dwarf" problem, or the lack of a large population of low metallicity stars, detected in the spectral energy distribution of ellipticals (Bressan et al. 1994; Worthey et al. 1996);

- the high fraction of white dwarfs (Bica et al. 1996);

- both the colour-magnitude relation and the trends in $\alpha$-enhancement with mass at the same time, thereby overcoming the well-known dichotomy between the "classic" and "inverse" GW scenario (Matteucci 1992, 1994, 1997).

This last point is worth commenting further, as the modelling of the GW influences directly the predictions concerning the ICM. GW models of elliptical galaxies with a constant IMF (whether Salpeter or more top-heavy) face the following puzzle. The colour-magnitude relation suggests that GWs occur later in more massive ellipticals than in smaller ones, so that star formation and chemical enrichment proceed longer and the stellar population reaches redder colours in more luminous objects. On the other hand, metallicity indices, if interpreted as abundance indicators, suggest that the $[\mathrm{Mg} / \mathrm{Fe}]$ ratio increases with galactic mass; this requires GWs to occur earlier in more massive galaxies, where SN II should dominate the chemical enrichment to make the resulting abundance ratios in stars $\alpha$-enhanced.

This dichotomy between the so-called "classic" and "inverse" GW scenario hampers predictions of the metal pollution of the ICM, since two competing sets of GW models are to be considered. It is therefore quite appealing, when we address the chemical enrichment of the ICM, that the variable IMF scenario described above can reproduce both observational constraints, with a unique set of models.

Chiosi (2000b) first analyzed the predicted metal pollution of the ICM when galaxy models with the PNJ IMF are adopted. To this purpose, he calculated multi-zone chemical models of ellipticals with the PNJ IMF.

The adoption of radial multi-zone models, rather than simple one-zone models, has in fact important consequences on the predicted enrichment of the ICM, as underlined first by Martinelli et al. (2000). When the radial structure of an elliptical galaxy is considered, with the corresponding gradients in density, colours etc., it turns out that the GW does not develop instantly over the whole galaxy, but it tends to set in earlier in the outskirts, where the potential well is shallower, and later in the central parts. This means that star formation and chemical enrichment proceed longer in the centre than in the outer regions (Tantalo et al. 1998; Martinelli et al. 1998), and the GW ejected from different galactic regions is metal enriched to different degrees.

The models calculated by Chiosi (2000b) account for this effect by dividing the galaxy into three zones: a central sphere where star formation and metal production is most efficient and lasts longer; an intermediate shell where the GW sets in earlier and the metal production proceeds to a lesser extent; 
an outer corona where the gas is expelled almost immediately, with virtually no star formation and chemical processing. This behaviour is the combined result of the shallower potential well when moving outward in the galaxy (as in standard models with a constant IMF) and of the varying $M_{\mathrm{p}}$ in the PNJ IMF when moving to outer, less dense regions; see Chiosi (2000b) for a detailed discussion.

For the sake of comparison, analogous models with the Salpeter IMF were also calculated, with mass range [0.18-120] $M_{\odot}$, as suited to model elliptical galaxies (Tantalo et al. 1998).

In all these models (Chiosi et al. 1998; Chiosi 2000b) the metal production and recycling, and the resulting abundances of the GW ejecta, are followed with the chemical evolution network developed by Portinari et al. (1998). Chemical yields of massive stars were derived from the stellar tracks of the Padua group for the pre-supernova phases, and then linked to the SN II models by Woosley \& Weaver (1995), rescaled to the same core masses. Yields for low and intermediate mass stars were taken from Marigo et al. (1996, 1998), however these are of minor importance in the present work: for the GW ejecta and the enrichment of the ICM, most important are iron and $\alpha$-elements, produced by supernovæ. The chemical network also includes type Ia SN, which are important iron contributors, adopting the recipe by Greggio \& Renzini (1983) for the rate, and the ejecta from the W7 model by Thielemann et al. (1993). We refer the reader to the original papers for further details on the chemical network.

\subsection{Galactic ejecta: PNJ vs. Salpeter IMF}

For a better understanding of the results concerning the chemical evolution of the ICM, let's first inspect the predicted GW ejecta of the galactic models when the variable IMF or the Salpeter IMF are adopted in turn.

A (proto) galaxy of initial baryonic mass $M$ formed at redshift $z_{\text {for }}$ ejects a mass $E_{\mathrm{GW}}\left(M, z_{\text {for }}\right)$ of gas as $\mathrm{GW}$, while a mass

$R_{\mathrm{gal}}\left(M, z_{\text {for }}\right)=M-E_{\mathrm{GW}}\left(M, z_{\text {for }}\right)$

remains as the baryonic component of the galaxy we "see today", that is as the stars (and remnants) of the final galaxy. For galactic models with the Salpeter IMF, the various quantities depend only on $M$ and not on $z_{\text {for }}$, as there are no temperature effects on the IMF in that case. For the purpose of the chemical evolution of the ICM, $R_{\text {gal }}$ can be viewed as a "galactic remnant", passively subtracting mass from further chemi$\mathrm{cal} /$ galactic processing, analogous to stellar remnants in chemical models of individual galaxies. The gas shed by long-lived stars after the GW episode is supposed to remain in the galaxy and not contribute further to the enrichment of the ICM.

In Fig. 1 we plot the mass fraction of gas ejected in the GW

$f_{\mathrm{GW}}=\frac{E_{\mathrm{GW}}}{M}$

and the complementary mass fraction locked into the "galactic remnant",

$f_{\text {gal }}=\frac{R_{\mathrm{gal}}}{M}=1-f_{\mathrm{GW}}$

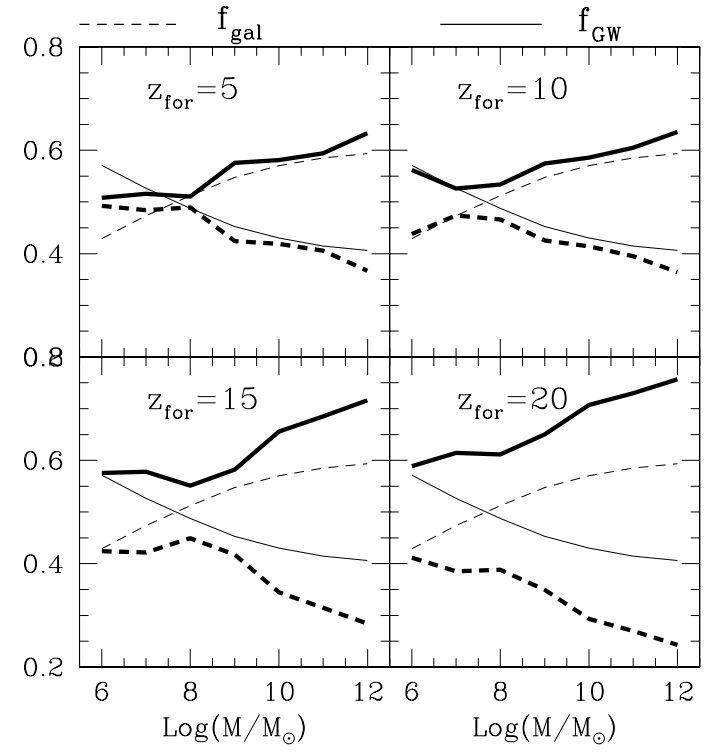

Fig. 1. Mass fractions in GW and in remaining galaxy as a function of the initial (proto)galactic mass $M$, for four different redshifts of formation. Thick lines: galactic models with the PNJ IMF; thin lines: galactic models with the Salpeter IMF.

for galactic models with the variable PNJ IMF and for models with the Salpeter IMF - thick and thin lines, respectively. Mass fractions refer to the total initial baryonic mass of the (proto)galaxy. The amount of ejected gas is larger in the case of the PNJ IMF, since less mass is locked into low-mass stars thanks to the high $M_{\mathrm{p}}$ in the early galactic phases. In particular, with the PNJ IMF the mass ejected as GW is always larger than that locked up in the galaxy. The difference with the Salpeter case gets sharper for larger (proto)galactic masses, and for higher redshifts of formation (as already mentioned, models with the Salpeter IMF bear in fact no dependence on the redshift of formation). It is worth underlining here the following "inverse" behaviour of the models with the PNJ IMF with respect to what is generally found from models with a constant IMF. According to the general consensus, larger galaxies store a larger fraction of their mass into stars and eject a lower fraction of gas in the $\mathrm{GW}$, while smaller galaxies, due to their shallower potential wells, are more efficient in wind ejection. This trend is indeed evident in the Salpeter galactic models of Fig. 1. The models with the PNJ IMF, on the other hand, show quite the opposite behaviour: larger galaxies eject a larger fraction of their initial mass in the wind and lock a lower fraction into stars, due to the higher peak mass that characterizes them in the early phases. This IMF effect overwhelms their deeper potential wells, and the trend becomes stronger and stronger with increasing redshift of formation. There are in fact arguments advocating large baryon losses from galaxies in general (Silk 2003).

The masses of iron and oxygen, respectively, ejected in the $\mathrm{GW}$ are $E_{\mathrm{GW}, \mathrm{Fe}}$ and $E_{\mathrm{GW}, \mathrm{O}}$, so that the metal abundances of the $\mathrm{GW}$ are given by:

$X_{\mathrm{GW}, \mathrm{Fe}}=\frac{E_{\mathrm{GW}, \mathrm{Fe}}}{E_{\mathrm{GW}}} \quad X_{\mathrm{GW}, \mathrm{O}}=\frac{E_{\mathrm{GW}, \mathrm{O}}}{E_{\mathrm{GW}}}$. 


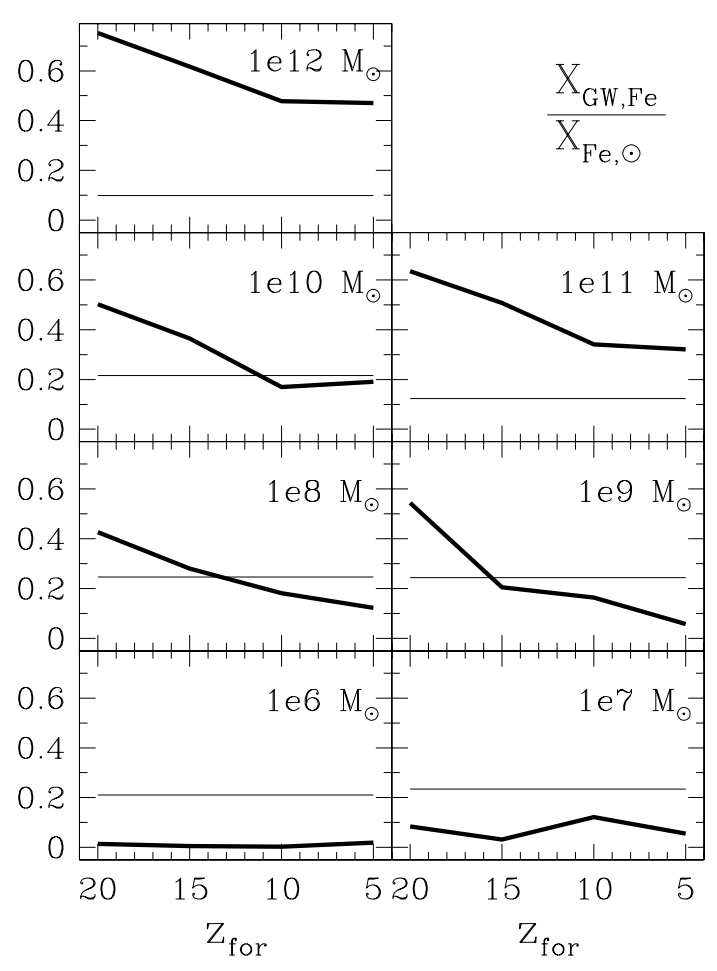

Fig. 2. Iron abundance (in solar units) of the GW, for galaxies of initial (baryonic) mass $M$ as indicated in each panel, and as a function of the redshift of formation. Thick lines: galactic models with the PNJ IMF; thin lines: models with the Salpeter IMF (redshift independent).

The quantities $E_{\mathrm{GW}}, E_{\mathrm{GW}, \mathrm{Fe}}, E_{\mathrm{GW}, \mathrm{O}}$ for a grid of values of $\left(M, z_{\text {for }}\right)$ are tabulated in Chiosi $(2000$, his Table 4, detailing the contribution of the three different shells).

Figure 2 shows the iron abundance $X_{\mathrm{GW}, \mathrm{Fe}}$ in the gas ejected as GW, again comparing the Salpeter IMF (thin lines) and the PNJ IMF case (thick lines). In most cases, the galactic ejecta in the PNJ models are more metal-rich than in the Salpeter case, up to a factor of five or more in the case of the most massive galaxies, and for high redshifts of formation. In the PNJ models, in fact, more galactic gas gets recycled through massive stars, effective metal contributors, and less mass gets locked into low-mass stars, before the GW occurs.

From the trends described above, we expect that models of ellipticals with the PNJ IMF predict a more efficient metal enrichment of the ICM, and a higher fraction of its gas originating from GWs, with respect to "standard" models. The first results in this respect were discussed by Chiosi (2000b).

\section{3. $M / L$ ratio and $I M L R$ of galaxies: $P N J$ vs. Salpeter}

A popular way of measuring the efficiency of metal production of cluster galaxies is the so-called iron mass to light ratio (IMLR), introduced by Ciotti et al. (1991); Renzini et al. (1993). This quantity is analogous to the "global yield" in chemical evolution models (Tinsley 1980; Pagel 1997), defined as the ratio of the global amount of metals produced by a generation of stars to the mass that remains locked in remnants plus low-mass, ever living stars. In the case of clusters, the mass locked in stars, i.e. in the stellar component of galaxies, is replaced by their luminosity (in the $B$-band), which is directly measurable, while the estimate of the mass would be indirect and would require some assumption about the $M / L$ ratio of cluster galaxies. Renzini $(1997,2000)$ estimates, for the $I M L R$ of the ICM:

$\frac{M_{\mathrm{ICM}}^{\mathrm{Fe}}}{L_{B}}=(0.02 \pm 0.01) h_{50}^{-\frac{1}{2}}$

where $M_{\mathrm{ICM}}^{\mathrm{Fe}}$ is the mass of iron in the ICM gas, $L_{B}$ is the global luminosity of cluster galaxies, and $h_{50}$ is the Hubble constant in units of $50 \mathrm{~km} \mathrm{~s}^{-1} \mathrm{Mpc}^{-1}$. The IMLR appears to be quite constant among rich clusters (Renzini 1997). More recent estimates of the IMLR taking into account the existence of radial gradients of iron abundance in the ICM and considering gas masses, iron masses and luminosities within the same radius, have slightly lowered the estimate (Finoguenov et al. 2000, 2001):

$\frac{M_{\mathrm{ICM}}^{\mathrm{Fe}}}{L_{B}}=(0.01-0.015) h_{50}^{-\frac{1}{2}}$.

These latter estimates have the advantage to be carried out consistently over the same cluster volume. Metallicities are in fact typically measured at most within half of the virial radius (Finoguenov et al. 2000, 2001; De Grandi \& Molendi 2001; Irwin \& Bregman 2001); measurements hardly reach $r_{500}$, which is typically $63 \%$ of $r_{200}$, and in terms of cluster mass $M_{500} \sim 0.63 M_{200}$ as well (see Reiprich \& Böhringer 2002) ${ }^{1}$. For the sake of the chemical enrichment what matters is the mass averaged metallicity, obtained by convolving metallicity distributions with gas profiles within the same radius, and the $I M L R$ should also be evaluated from the gas, metals and galactic luminosity within the same radius (as in Finoguenov et al. $2000,2001,2003$ ). Since the observed metallicities probe only half of the cluster mass, considering them representative of the whole gas mass (derived from the gas profile extended out to the virial or Abell radius) is a significant extrapolation, especially in view of recent results on gradients: relaxed clusters with cooling flows systematically show iron abundance gradients (also besides the central peak) and even the metallicity distribution in non-cooling flow clusters, though consistent with being uniform, is better fitted with a negative gradient (De Grandi \& Molendi 2001).

The IMLR could also be overestimated in the presence of a substantial intra-cluster stellar populations. Most studies limit this diffuse population to a $10-20 \%$ of the total stellar content, but values up to $40 \%$ have been recently suggested (Arnaboldi et al. 2002).

Finally, we remind that red or NIR bands are a better probe of the actual star mass in galaxies, for they are less sensitive to recent sporadic star formation and more to the old underlying population. Hence the IMLR would be a better probe of the real "yield" of galaxies, if it were expressed in terms of the $R$ to $K$ band luminosity. This is becoming possible nowadays, as

\footnotetext{
1 We indicate, as customary, with $r_{N}$ the radius corresponding to an overdensity $N$ times larger than the average background density; $r_{200}$ is usually identified with the virial radius. $M_{N}$ indicates the mass enclosed within $r_{N}$.
} 


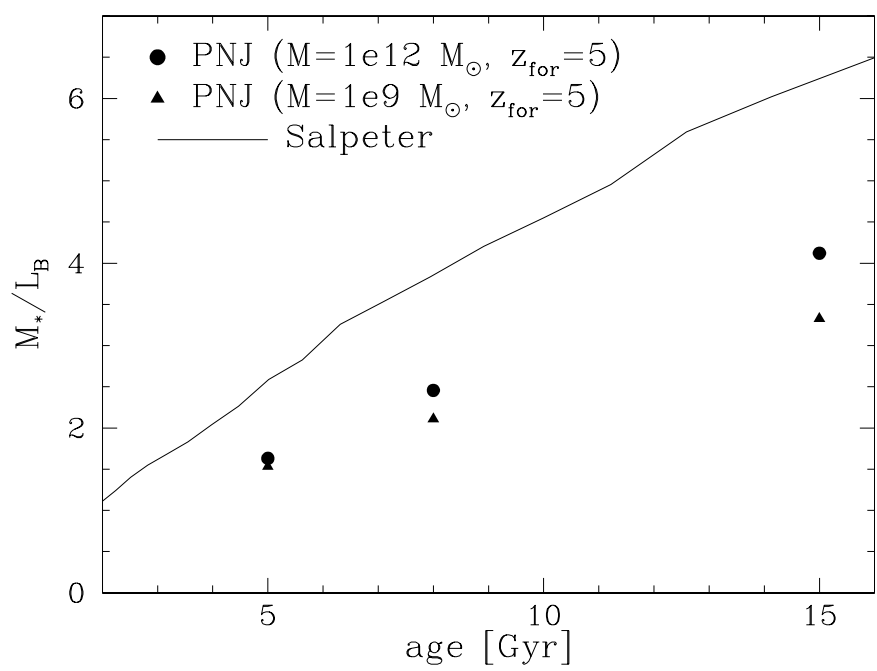

Fig. 3. The $M / L$ ratio in the $B$ band for living stars in the PNJ galaxies (dots and triangles, two example models, see legend) and in the Salpeter galaxies (thin line; mass limits for the Salpeter IMF are [0.18-120] $\left.M_{\odot}\right)$.

cluster LFs in red or IR bands are presently becoming available (Mobasher et al. 2003; Driver et al. 1998; Mobasher \& Trentham 1998; Trentham \& Mobasher 1998; De Propris et al. 1999; Andreon \& Pelló 2000; Andreon \& Cuillandre 2002); see in fact Lin et al. (2003)

While these developments are certainly interesting for the future, here we will consider as our observational constraint the "canonical" value of the IMLR in the $B$ band given by (1).

Before modelling the cluster and its $I M L R$ as a whole, it is worth considering what is the $I M L R$ of the galactic wind of individual model galaxies:

$I M L R_{\mathrm{GW}}=\frac{E_{\mathrm{GW}, \mathrm{Fe}}}{L_{B}}$

comparing the PNJ IMF to the Salpeter IMF case. The GW ejecta $E_{\mathrm{GW}, \mathrm{Fe}}$ have been discussed above, now we discuss the $B$-band luminosities $L_{B}$ of our galaxies. A galaxy, or "galactic remnant" $R_{\text {gal }}$ left over after the galactic wind, consists of three baryonic components: the living stars producing the galactic luminosity, the dark stellar remnants and the gas shed by stars after the GW. The first component progressively loses mass in favour of the other two; namely, within $R_{\text {gal }}$ the fraction $F_{*}$ of living stars decreases with time (Fig. 4).

Comparing the PNJ and the Salpeter models, two contrasting effects contribute to determine the final luminosity of a galaxy. With the PNJ IMF, in the early galactic phases the typical stellar mass was skewed to higher values and less material was locked into low mass, very low luminosity stars. As a consequence, the living stars are on average more massive and more luminous in the PNJ models than in the Salpeter models, so that their typical $M / L$ ratio is lower (Fig. 3). On the other hand, with the PNJ IMF more mass went into remnants in the early, top-heavy star formation phases, and the gas restitution fraction is higher (even after the GW) because of the lower number of ever-living low-mass stars; as a consequence, living stars often represent a lower mass fraction of the final galaxy

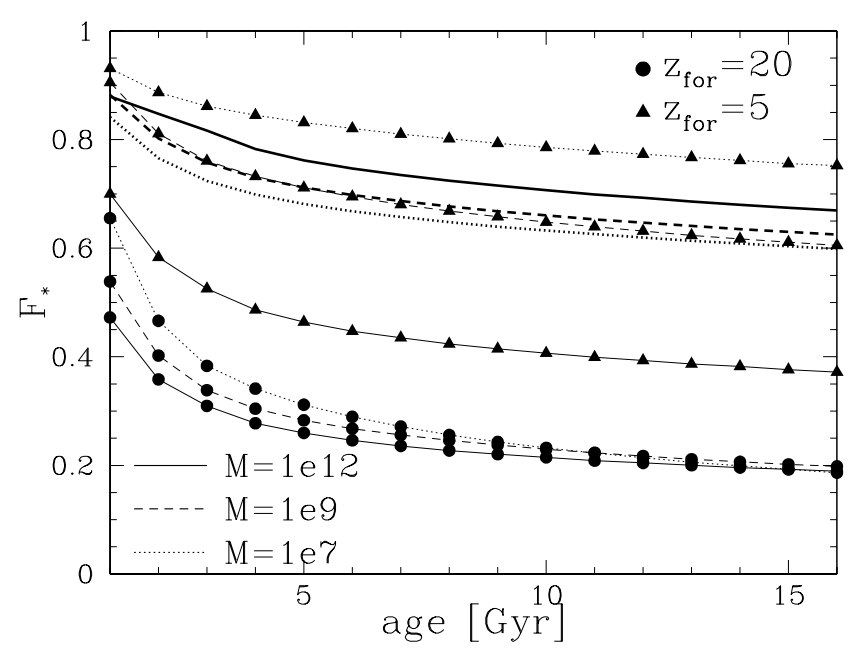

Fig. 4. Fraction of living stars within the "galactic remnant" $R_{\text {gal }}$, decreasing with age in favour of remnants and ejected gas. PNJ models for three initial (proto)galactic masses and two redshifts of formation shown for the sake of example (see legend). Thick lines with no dots correspond to the Salpeter models.

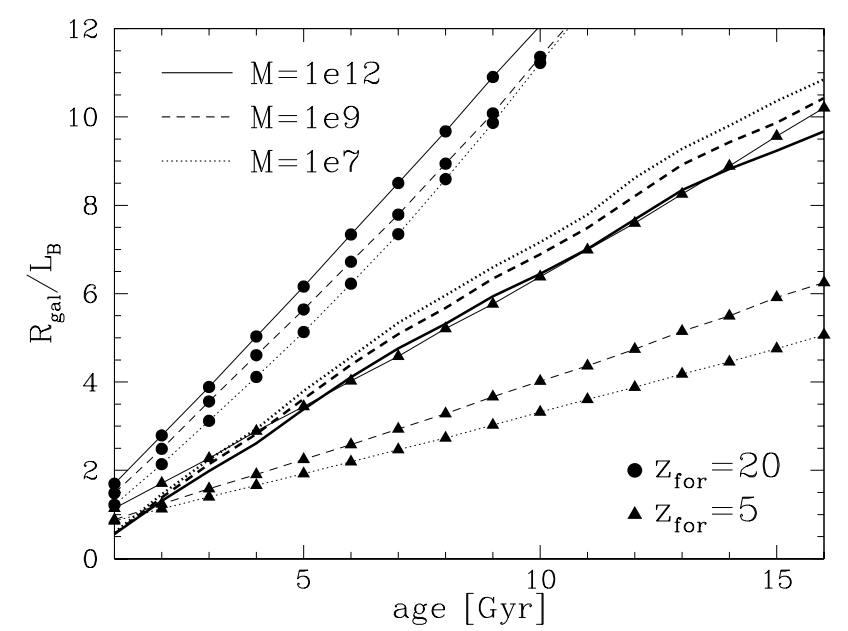

Fig. 5. $M / L$ ratio of the global "galactic remnant" $R_{\text {gal }}$ (stars+remnants+gas ejected after the $\mathrm{GW}$ ), as a function of age. Symbols as in Fig. 4.

in the PNJ models, especially for high redshifts of formation and/or large masses (Fig. 4). Globally, the total $M / L$ ratio of the galaxies, or "galactic remnants" made of stars+remnants+gas (ejected by stars after the GW), is shown in Fig. 5. With respect to the Salpeter models, models with the PNJ IMF show a composite behaviour: at low redshifts of formation $\left(z_{\text {for }} \lesssim 10\right.$ ) they have lower $M / L$ ratios, for the effect of having more luminous stars prevails; at high redshifts of formation, they have higher $M / L$ ratios, for the effect of the larger amount of remnants prevails.

Finally, Fig. 6 displays the $I M L R_{\mathrm{GW}}$ of the galaxies (Eq. (2)) as a function of the initial mass $M$ of the (proto)galaxy and of its redshift of formation $z_{\text {for }}$; thick lines are for galaxies with the PNJ IMF, thin lines are for the Salpeter case. Notice that $I M L R_{\mathrm{GW}}$ depends on $z_{\text {for }}$ also for the Salpeter case, for although $E_{\mathrm{GW}, \mathrm{Fe}}$ is independent of redshift with the Salpeter IMF, 


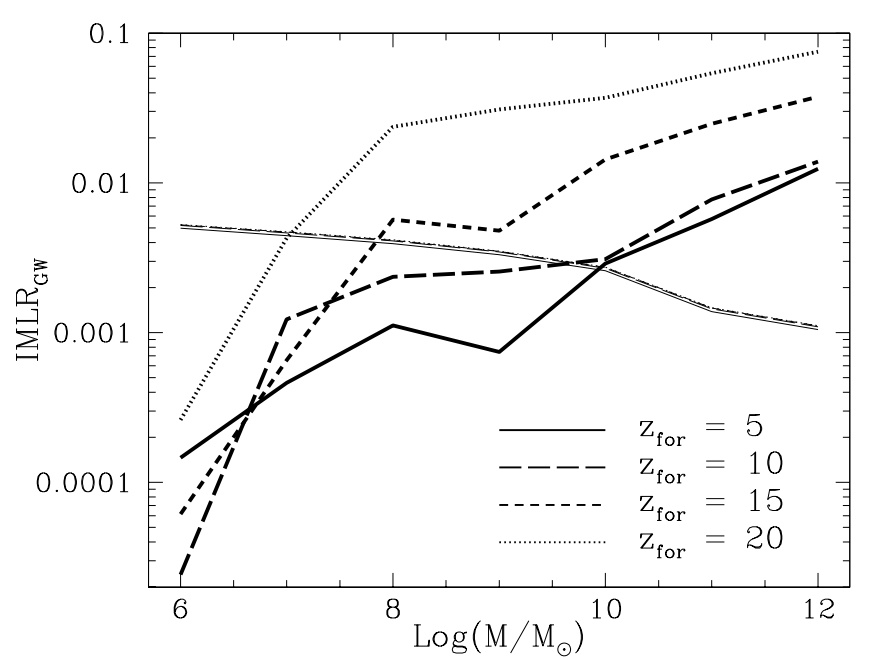

Fig. 6. The $I M L R$ in the GW for model galaxies with the PNJ IMF (thick lines) and the Salpeter IMF (thin lines).

$L_{B}$ decreases due to increasing galactic age at increasing $z_{\text {for }}$ (the effect is however negligible between $z_{\text {for }}=20$ and 5).

The trend of $I M L R_{\mathrm{GW}}$ with mass $M$ is opposite in the two cases, reflecting the effects already commented upon with Figs. 1 and 2. At small masses, models with the Salpeter IMF eject more gas and metals in the $\mathrm{GW}$, retaining less mass in stars and having a lower final luminosity; their $I M L R_{\mathrm{GW}}$ is thus higher than that of the PNJ IMF models. At large masses, say $M \gtrsim$ a few $10^{10} M_{\odot}$, the behaviour is reversed and it is the galactic models with the PNJ IMF that eject a higher amount of metals relative to the luminous mass retained in the "galactic remnant". For $z_{\text {for }}<5$, GW ejecta are the same as in the $z_{\text {for }}=5$ case, as the background temperature has by then dropped to low values and there is no further evolution on its basic effects on the IMF. Therefore for $z_{\text {for }}<5$, for both IMFs the corresponding $I M L R_{\mathrm{GW}}$ changes (decreases) just because of the effects of increasing $L_{B}$ at younger ages.

Most important for the sake of the chemical enrichment of the ICM, notice that the Salpeter models for all masses have an intrinsic $I M L R_{\mathrm{GW}}<0.01$, i.e. always lower than what is observed in clusters (Eq. (1)). We can thus anticipated that model galaxies with the Salpeter IMF are incapable of reproducing the high IMLR observed in clusters. On the contrary, models with the PNJ IMF at high masses and/or for high redshifts of formation do reach $I M L R_{\mathrm{GW}} \geq 0.01$.

To predict the $I M L R$ in the cluster as a whole, we need to convolve the respective $I M L R_{\mathrm{GW}}$ with the Galactic Formation History of the cluster; we will see (Sect. 6) that models with the PNJ IMF yield a higher global IMLR, in better agreement with observations. This is due to the fact that small galaxies, although dominating the LF in number, actually represent a minor contribution in mass, so that the main role in the ICM pollution is played by galaxies with $M \gtrsim 10^{10} M_{\odot}$ (see also Thomas 1999). For the latter type, galactic models with the PNJ IMF have in fact an $I M L R_{\mathrm{GW}}$ of the order of 0.01-0.02, comparable to the typical value $\sim 0.01$ for clusters.

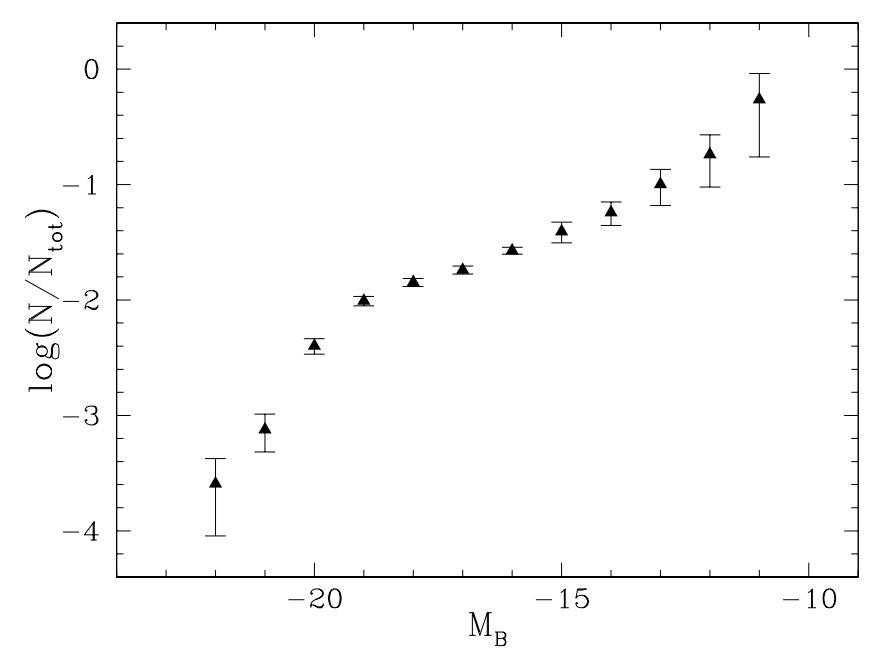

Fig. 7. The observed $B$-band luminosity function of cluster galaxies by Trentham (1998), in relative frequency.

\section{Global cluster results: The monolithic approach}

As mentioned in Sect. 2, the most popular way to calculate the metal content of the ICM of a cluster on the basis of its population of elliptical galaxies is to develop a grid of galactic models and integrate their GW ejecta over the observed LF, a method first introduced by Matteucci \& Vettolani (1988). In this approach, all the ellipticals in the cluster are assumed to be coeval, hence we will call this the "monolithic" approach.

In this section we adopt the monolithic method, with the PNJ and the Salpeter galactic models in turn. Our reference LF is the observed $B$-band LF by Trentham (1998), since it is very deep (down to magnitude $M_{B}=-11$ ) and since the observed $I M L R$ is also referred to the $B$-band luminosity (see Sect. 3.3). This LF, displayed in Fig. 7, is a weighted mean of the LFs of 9 clusters at low redshift $(z<0.2)$. Besides showing the standard exponential cut-off at bright magnitudes $\left(M_{B}<-20\right)$, characteristic of a Schechter (1976) function, this LF also steepens at the faint end $\left(M_{B}>-14\right)$, that is in the regime of dwarf galaxies.

The luminosity evolution of the "galactic remnants" is known as a function of the initial (proto)galactic mass $M$ and of the redshift of formation $z_{\text {for }}$ (Sect. 3.3). As to the relation between the redshift $z_{\text {for }}$ and the corresponding age of the galaxy, we adopt a flat $\Lambda$ Cold Dark Matter $(\Lambda \mathrm{CDM})$ cosmology with $\Omega_{\mathrm{M}}=0.3, \Omega_{\Lambda}=0.7$ and $H_{0}=65 \mathrm{~km} \mathrm{~s}^{-1} \mathrm{Mpc}^{-1}$. Once a value for $z_{\text {for }}$ is fixed, each luminosity bin of the LF corresponds to some initial galactic mass $M$, and we sum the contributions of the different bins to estimate the global amounts of ejected gas and metals.

The GW ejecta of the PNJ models are quite sensitive to the exact epoch (redshift) of formation of the individual galaxies, as discussed in Sect. 3.2, and we perform the exercise for $z_{\text {for }}=5,10,13,15,20$. (The case $z_{\text {for }}=13$ is obtained by interpolation in the grid of galactic models.) For the Salpeter IMF, instead, computing the case $z_{\text {for }}=5$ is sufficient because the GW ejecta are fixed, and the age and luminosity differences for $z_{\text {for }}>5$ are negligible (Fig. 6). 
Table 1. Results for integrated quantities for the cluster, in the monolithic approach. Column 1: IMF of model galaxies. Column 2: redshift of formation of galaxies. Column 3: global IMLR for the cluster. Column 4: global $M / L$ ratio for cluster galaxies (ratio between the global mass in "galactic remnants" and the global B-luminosity). Column 5: ratio between the mass of gas ejected as GW and the global $B-$ luminosity of cluster galaxies. Column 6: ratio between the mass in gas ejected as GW and the mass in "galactic remnants". Column 7: iron abundance with respect to solar in the gas ejected as GW. Column 8: fraction of the global iron production contributed by SN Ia. Column 9: [O/Fe] ratio in the gas ejected as GW (and hence in the ICM). Column 10: "dilution factor" necessary to recover the observed $I C M L R=M_{\mathrm{ICM}} / L_{\text {tot }}=37 M_{\odot} / L_{\odot}$ (see text). Column 11: ratio between the total mass of ICM gas and the mass in galaxies. Column 12: average iron abundance in the ICM gas.

\begin{tabular}{crrrrrrrrrrrr}
\hline \hline IMF & $z_{\text {for }}$ & \multirow{2}{*}{$I M L R$} & $\frac{M_{\mathrm{gal}}}{L_{\mathrm{tot}}}$ & $\frac{M_{\mathrm{GW}}}{L_{\mathrm{tot}}}$ & $\frac{M_{\mathrm{GW}}}{M_{\mathrm{gal}}}$ & $\frac{X_{\mathrm{Fe}}, \mathrm{GW}}{X_{\mathrm{Fe}, \odot}}$ & $f_{\mathrm{SNIa}}$ & {$[\mathrm{O} / \mathrm{Fe}]$} & $\frac{M_{\mathrm{ICM}}}{M_{\mathrm{GW}}}$ & $\frac{M_{\mathrm{ICM}}}{M_{\mathrm{gal}}}$ & $\frac{X_{\mathrm{Fe}}, \mathrm{ICM}}{X_{\mathrm{Fe}, \odot}}$ \\
\hline PNJ & 5 & 0.007 & 7.2 & 11.1 & 1.54 & 0.358 & 0.53 & -0.08 & 3.3 & 5.1 & 0.11 \\
PNJ & 10 & 0.009 & 8.2 & 13.0 & 1.59 & 0.376 & 0.54 & -0.07 & 2.8 & 4.5 & 0.13 \\
PNJ & 13 & 0.018 & 10.6 & 21.1 & 1.99 & 0.477 & 0.47 & +0.07 & 1.8 & 3.5 & 0.27 \\
PNJ & 15 & 0.029 & 12.5 & 28.9 & 2.31 & 0.562 & 0.43 & +0.16 & 1.3 & 2.9 & 0.44 \\
PNJ & 20 & 0.065 & 17.4 & 50.6 & 2.91 & 0.714 & 0.36 & +0.21 & - & - & - \\
Salpeter & 5 & 0.002 & 8.6 & 6.2 & 0.72 & 0.143 & 0.65 & -0.09 & 6.0 & 4.3 & 0.02 \\
\hline
\end{tabular}

Results for global integrated quantities with the PNJ and the Salpeter models are listed in Table 1 . The 3rd column shows the global IMLR for the cluster. The Salpeter models fail to reproduce the observed IMLR by an order of magnitude or so. The PNJ models perform much better, with an iron production and hence an $I M L R$ increasing with redshift of formation, as expected from Sect. 3.3. For $z_{\text {for }}=10-13$ the $I M L R$ falls in the observed range (Eq. (1)), while models with $z_{\text {for }}>13$ have too large an iron production with respect to observations.

\subsection{The contribution of type la supernovæ}

At increasing redshift of formation, the PNJ IMF is more and more skewed toward massive stars in the early phases, implying a lower and lower ratio of the number of SN Ia vs. SN II. Column 8 in Table 1 reports the fraction of the global iron produced and ejected into the ICM due to SN Ia; as expected, it decreases with increasing redshift, from 55 to $35 \%$. The Salpeter IMF has a larger contribution $(65 \%)$ from $\mathrm{SN}$ Ia than any case of the PNJ IMF.

The same trends are reflected in the $[\mathrm{O} / \mathrm{Fe}]$ ratio of the ejected gas (9th column in the table), increasing with redshift. Within the present uncertainties about the $[\alpha / \mathrm{Fe}]$ ratio in the ICM (Sect. 1), all of the values for $[\mathrm{O} / \mathrm{Fe}]$ in Table 1 are acceptable. For the "favoured" cases (on the base of the $I M L R$ ) of $z_{\text {for }}=10-13$, the resulting $[\mathrm{O} / \mathrm{Fe}]$ ratio is close to solar.

One concern when computing the iron production and ejection from galactic models is the uncertainty in the rate of SN Ia, due to the poorly known evolution of the progenitors. As a consequence, one may wonder if the high iron abundances in the ICM can be reproduced by adopting a higher rate of SN Ia rather than a top-heavy or non-standard IMF. However, the $[\mathrm{O} / \mathrm{Fe}]$ ratios we obtain indicate that the relative contribution of SN Ia vs. SN II predicted for the ICM are grossly correct.

With the Salpeter IMF and standard GWs models of elliptical galaxies, the predicted contribution to the iron enrichment of the ICM is short by 5-10 times with respect to the observed IMLR. If we were to compensate for this discrepancy by adjusting the SN Ia rates to the required level - or by assuming that later iron production from SN Ia escapes the galaxy after the main $\mathrm{GW}$ episode - the corresponding $[\mathrm{O} / \mathrm{Fe}]$ ratios would decrease by $0.7-1$ dex, by far too low with respect to observations.

\subsection{The problem of dilution}

Besides the amount of ejected metals as traced by the IMLR, an important question concerns the global gas mass ejected from galaxies as GW: can this account for the whole of the ICM, or conversely most of the ICM is primordial gas that was never involved in galaxy formation? The observational constraint here is the global amount of ICM gas vs. the mass in galaxies; unfortunately, this quantity is relatively poorly determined. In literatures, values can be found in the range $M_{\mathrm{ICM}} / M_{\mathrm{gal}}=2-20$; a closer inspection reveals that this large range can be mostly imputed to different assumptions for the $M / L$ ratios for galaxies. In fact, while the mass in the ICM can be estimated directly from X-ray observations, the mass in galaxies is estimated from their global luminosity, usually in the $B$-band, and assuming a $M / L$ ratio. We remark here that for the chemical enrichment of the ICM, what is meaningful is the baryonic mass in galaxies, not their global mass or $M / L$ ratio inclusive of the dark halo component. The real observable is $M_{\mathrm{ICM}} / L_{\mathrm{B}}$, and with respect to this quantity results in literature are much more homogeneous.

From the study of David et al. (1990) of Hydra A one finds $M_{\mathrm{ICM}} / L_{V}=33 h_{50}^{-\frac{1}{2}} M_{\odot} / L_{\odot}$; the typical colours of ellipticals correspond to $L_{V} \sim 1.3 L_{B}$ and the above entry translates into:

$\frac{M_{\mathrm{ICM}}}{L_{B}}=30 h^{-\frac{1}{2}} \frac{M_{\odot}}{L_{\odot}}$.

Arnaud et al. (1992) assume $L_{V} \sim 1.2 L_{B}$ for the typical colours of $\mathrm{E}$ and $\mathrm{S} 0$ galaxies, and consequently derive for their clusters $M_{\mathrm{ICM}} / L_{V,(\mathrm{E}+\mathrm{S} 0)} \sim(20-50) h_{50}^{-\frac{1}{2}}$, with an average of $36 h_{50}^{-\frac{1}{2}}$ (see also Elbaz et al. 1995), corresponding again to:

$\left\langle\frac{M_{\mathrm{ICM}}}{L_{B}}\right\rangle \sim 30 h^{-\frac{1}{2}} \frac{M_{\odot}}{L_{\odot}} \quad$ with a range $(17-42) h^{-\frac{1}{2}}$

The analysis of the Coma cluster by White et al. (1993) yields as well:

$\frac{M_{\mathrm{ICM}}}{L_{B}} \sim 30 h^{-\frac{1}{2}} \frac{M_{\odot}}{L_{\odot}}$ 
Cirimele et al. (1997) estimate $M_{\mathrm{ICM}} / M_{\text {gal }}$ for their sample under the assumption of a typical $M / L_{V}=8 M_{\odot} / L_{\odot}$ for elliptical galaxies; their tabulated values correspond to $M_{\mathrm{ICM}} / L_{V}$ in the range 8.5-37.6 with an average value of $21 M_{\odot} / L_{\odot}$. Taking again a typical $L_{V} \sim 1.3 L_{B}$ for ellipticals, and considering that Cirimele et al. assumed $H_{0}=50$, their data imply:

$$
\left\langle\frac{M_{\mathrm{ICM}}}{L_{B}}\right\rangle \sim 19 h^{-\frac{1}{2}} \frac{M_{\odot}}{L_{\odot}} .
$$

In the sample by Roussel et al. (2000), the average over all the objects is:

$$
\left\langle\frac{M_{\mathrm{ICM}}}{L_{B}}\right\rangle \sim 31 h^{-\frac{1}{2}} \frac{M_{\odot}}{L_{\odot}}
$$

while for the hot clusters only, a somewhat higher value is obtained:

$$
\left\langle\frac{M_{\mathrm{ICM}}}{L_{B}}\right\rangle \sim 44 h^{-\frac{1}{2}} \frac{M_{\odot}}{L_{\odot}}
$$

although it depends on the volume probed: $M_{\mathrm{ICM}} / L_{B}=27,35$ or $44 h^{-\frac{1}{2}} M_{\odot} / L_{\odot}$, for $r<r_{2000}, r_{500}$ or $r<r_{200}$ respectively, since the gas distribution is generally more extended than the galaxy distribution.

Finoguenov et al. (2003) indicate a typical value of $M_{\mathrm{ICM}} / L_{B} \sim 30$ for hot clusters within $0.4 r_{100}$, adopting $H_{0}=70$, or:

$$
\frac{M_{\mathrm{ICM}}}{L_{B}} \sim 21 h^{-\frac{1}{2}} \text {. }
$$

In summary, data indicate a typical value of "intra-cluster mass to light ratio"

$I C M L R=\frac{M_{\mathrm{ICM}}}{L_{B}} \sim 30 h^{-\frac{1}{2}} \frac{M_{\odot}}{L_{\odot}}$

with somewhat lower values in the samples by Cirimele et al. (1997); Finoguenov et al. (2003) (though still growing with radius in the latter) and somewhat higher values in the sample by Roussel et al. (2000). Whether the fraction of hot vs. cold baryons, that is the efficiency of galaxy formation, increases noticeably with cluster temperature and richness is on the other hand still a matter of debate (Bryan 2000; Balogh et al. 2001; Lin et al. 2003).

We favour $M_{\mathrm{ICM}} / L_{B}$ as a constraint rather than $M_{\mathrm{ICM}} / M_{\mathrm{gal}}$, since the former is directly measured. Just as the IMLR is supposedly a more straightforward estimate of the efficiency of metal enrichment than the "classical" yield $M_{\mathrm{Fe}} / M_{*}$ (see Sect. 3.3), the ICMLR is a more direct constraint than the "classical" gas fraction $M_{\mathrm{ICM}} / M_{*}$ because, like the $I M L R$, it is independent of a priori assumptions on the $M / L$ ratio of galaxies. For our present favoured value of $h=0.65$, we regard $I C M L R \sim 37 M_{\odot} / L_{\odot}$ as our constraint for the total amount of ICM gas in clusters.

In the last three columns of Table 1 we give the dilution factors (i.e. the ratio between the total ICM gas mass and the mass provided by the GWs) necessary to recover the observed ICMLR, the corresponding ratio between ICM mass and mass in galaxies (the latter inclusive of stars, remnants and gas shed by stars after the GW) and the average metallicity in the global ICM. Typical dilution factors are in the range 2-3 for the PNJ models, and the ICM mass is typically 3-5 times the mass in galaxies.

In the case of $z_{\text {for }}=13$, that is the case with the best results for the IMLR, roughly half of the ICM is expected to come from GWs, its average metallicity is close to the observed characteristic value of 0.3 solar, and the ICM mass is 3.5 times the mass in galaxies. Galactic models with a redshift of formation $z_{\text {for }}>15$ are ruled out since they eject not only too much metals (as marked by the high IMLR) but also too much gas in the GW to be compatible with the observed ICMLR. We notice also that Salpeter models require a high degree of dilution (a factor of 6) and the corresponding metallicity in the ICM gas is a factor of 10 too low, as expected from the correspondingly low IMLR.

Our global $M / L$ ratio for galaxies (including all the baryonic components, living stars, remnants and gas shed by stars at late times) are in the range 7-12 for acceptable models with $z_{\text {for }} \leq 15$, and $\sim 8$ for the Salpeter models. Correspondingly, the observed $I C M L R=37 M_{\odot} / L_{\odot}$ implies that the mass in the ICM is 3-5 times the mass in galaxies. This is a factor of 2-3 lower than the widely quoted value of 10 derived by White et al. (1993) for $h=0.65$. The difference just stems from their much lower $M / L_{B}=6.4 h$, adopted on the basis of dynamical arguments; this is lower than what is expected for the typical stellar population in an old elliptical galaxy (cf. the $M / L$ ratio of Salpeter models). The difference is quite irrelevant for the problem discussed by White et al. (1993), that is the baryon fraction in clusters: the baryonic mass is dominated by the hot ICM gas mass anyways, and is not much affected by uncertainties in the mass in galaxies - though the latter might not be as negligible, as sometimes assumed. However, for the sake of the chemical enrichment of the ICM the effect is quite crucial. Adopting the ICM-to-galaxy mass ratio by White et al. (1993), Renzini (1997) estimates the ratio between the metals in the ICM and that locked in the stars of cluster galaxies to be $1.65 h^{-3 / 2}$, or $\sim 3$ for $h=0.65$. If the $M / L$ ratio in galaxies is higher, as required e.g. by stellar population models for ellipticals, the "metal balance" is much less skewed toward the ICM so that the amount of metals in the ICM becomes comparable to that in galaxies. This underlines the importance, for a consistent modelling of the chemical evolution of the ICM, to adopt the observed ICMLR as a constraint rather than some independently derived ICM-to-galaxy ratio relying on external assumptions on galactic $M / L$ ratios.

Finally, we remark that the actual ICM-to-galaxy mass ratio is important for the sake of explaining the "entropy floor" in low-T clusters, whether it requires strong supernova preheating or whether it is partly due to the removal of lowentropy gas by galaxy formation (Bryan 2000; Balogh et al. 2001; Tornatore et al. 2003). Interestingly, the recent cluster simulations by Valdarnini (2002) with star formation and selfconsistent chemical evolution, suggest that the metallicities of the ICM can be reproduced provided the IMF is top-heavy with respect to Salpeter, and the corresponding mass in cold baryons (galaxies, or "star particles" in the simulations) is large enough 


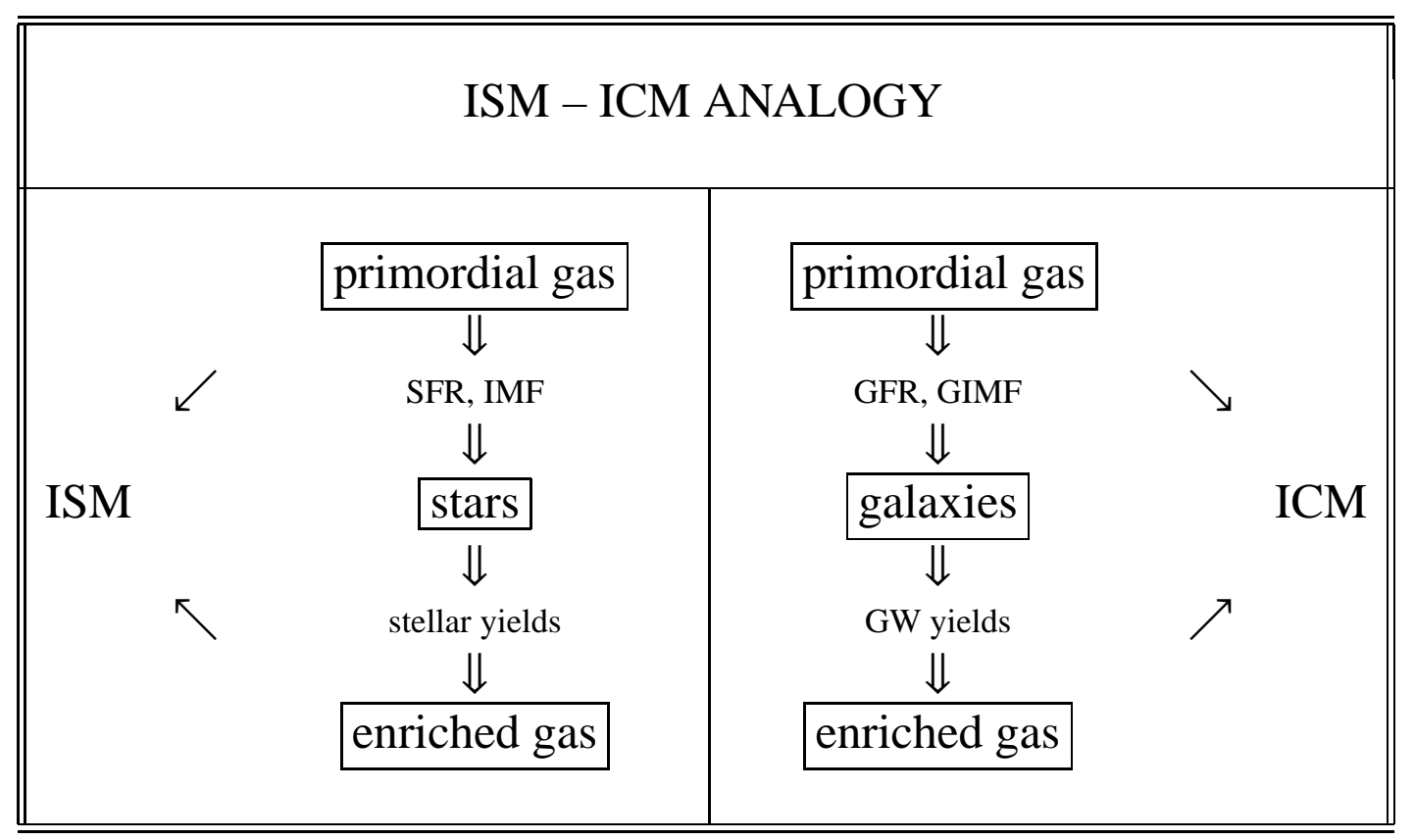

to favour the scenario of removal of low-entropy gas by Bryan (2000).

\section{A chemical model for the ICM evolution}

The monolithic approach of Sect. 4 assumes all the ellipticals in the cluster to be coeval, with an age of 13-14 Gyrs. This is a somewhat naïve approach in the light of current understanding of structure formation in the Universe. In the hierarchical scenario the typical size of virialized objects increases at decreasing redshift. There are various reasons to argue that this scenario, developed for the gravitationally dominating, collisionless dark matter, might not hold for baryons, whose evolution at galactic scales is probably decoupled from that of dark matter - the angular momentum problem of disc galaxies in cosmological simulations, the observational evidence of early formation of elliptical galaxies, the colour-magnitude relation of both disc and elliptical galaxies suggesting the stellar populations in larger objects to be older at odds with expectations from the hierarchical bottom-up assembly, etc. Hence it is possible that even in the hierarchical scenario, an old and roughly coeval stellar population of ellipticals can be accommodated, by suitable account of detailed baryon physics. Nevertheless, we explore now a picture in which the formation of cluster galaxies of different masses is redshift dependent; as the extreme alternative to the monolithic approach, we will assume that a galaxy forms at the epoch when the corresponding dark halo is predicted to virialize according to the Press-Schechter formalism. We develop a chemical model following the epoch of formation of the individual galaxies in the cluster, and evolve the overall system down to the present day, using the LF as a constraint a posteriori. This approach also allows, in principle, a more self-consistent description of the chemical evolution of the cluster and of its galactic population as a whole.

An improved modelling of the evolution of the cluster, taking into account that its galaxies may form at different redshifts, has been introduced by Chiosi (2000b), who replaced the usual integration over the LF with an integration over the Press \& Schechter (1974) mass function at different redshifts. On the same line, we developed a global, self-consistent chemical model for the cluster as a whole, following the simultaneous evolution of all of its components: the galaxies, the primordial gas, and the gas processed and re-ejected via GWs. The approach is also somewhat similar in spirit to the "cosmic chemical evolution" model by Pei \& Fall (1995), aimed at calculating the global evolution of large volumes of the Universe, populated by a variety of star forming objects.

Our chemical model for clusters is developed in analogy with the usual chemical models for galaxies. These latter are schematically conceived as follows (Tinsley 1980; Pagel 1997):

1. the gas present in the system (usually starting from primordial composition) transforms into stars according to some prescribed Star Formation Rate (SFR);

2. stars are thus formed, distributed according to the adopted IMF;

3. stars return part of their mass in the form of chemically enriched gas, according to the so-called stellar yields (prescriptions derived from stellar evolution and nucleosynthesis);

4. this chemically enriched gas mixes with the surrounding gas, causing the chemical evolution of the overall interstellar medium (ISM).

In a cluster, we are interested to model the chemical evolution of the ICM, and the objects responsible for its enrichment 
are the galaxies, via GWs. In analogy with the above scheme, therefore, our chemical model for the cluster is conceived as follows:

1. the primordial gas in the ICM gets consumed in time by galaxy formation according to some prescribed Galactic Formation Rate (GFR);

2. at each time (redshift) galaxies form distributed in mass according to a Galactic Initial Mass Function (GIMF), derived from the Press-Schechter mass function suited to that redshift;

3. galaxies restitute a fraction of their initial mass in the form of chemically enriched GWs, according to the adopted galactic models;

4. this enriched gas mixes with and causes the chemical evolution of the overall ICM, which includes the amount of primordial gas not yet consumed by galaxy formation and the gas re-ejected by galaxies in the GWs up to the present age.

The analogy between the two types of models is displayed in the scheme above.

Model equations parallel those of galactic chemical models, with the substitutions SFR $\rightarrow$ GFR, IMF $\rightarrow$ PressSchechter GIMF, stellar yields $\rightarrow \mathrm{GW}$ yields. The main assumptions at the base of the model are as follows.

- The model is one-zone, namely the cluster is treated as a single uniform compound of gas and galaxies, where the metal abundance of the gas evolves in time but is otherwise instantly mixed and homogeneous.

It is worth commenting here on how sensible one-zone models are for structures, like clusters, that in current cosmological theories form out of hierarchical accretion of subunits. We remind here that a one-zone chemical model contains no information about the spatial distribution of its components. Therefore, at high redshifts our "model cluster" can be considered simply as the sum of the subunits that will later merge and form it, irrespectively of whether the cluster has in fact formed or not, as a single bound gravitational structure. The chemical model at high redshifts just describes the average properties of the sum of the "parent subunits" of the cluster.

- The model is calculated assuming the Instantaneous Recycling Approximation (IRA), that is assuming that galaxies eject the corresponding GWs instantly, as soon as they are formed; this is a reasonable approximation as the timescales for the onset of the GW are generally short, less than 1 Gyr. The IRA could affect predictions at high redshifts, where a time-span of a few $10^{8} \mathrm{yr}$ corresponds to a sizeable gap in redshift; but up to redshift $z \sim 1$, where observational data on ICM abundances are available, the effect is minor.

Later on, the model might be improved in this respect, by taking into account the actual delay between the formation of a galaxy and the time when its GW is expelled; but as it is always better to start with the simplest possible assumptions, we adopt the IRA for the time being.

Notice however that the individual galactic models and their GW yields are not calculated in the IRA, but with a
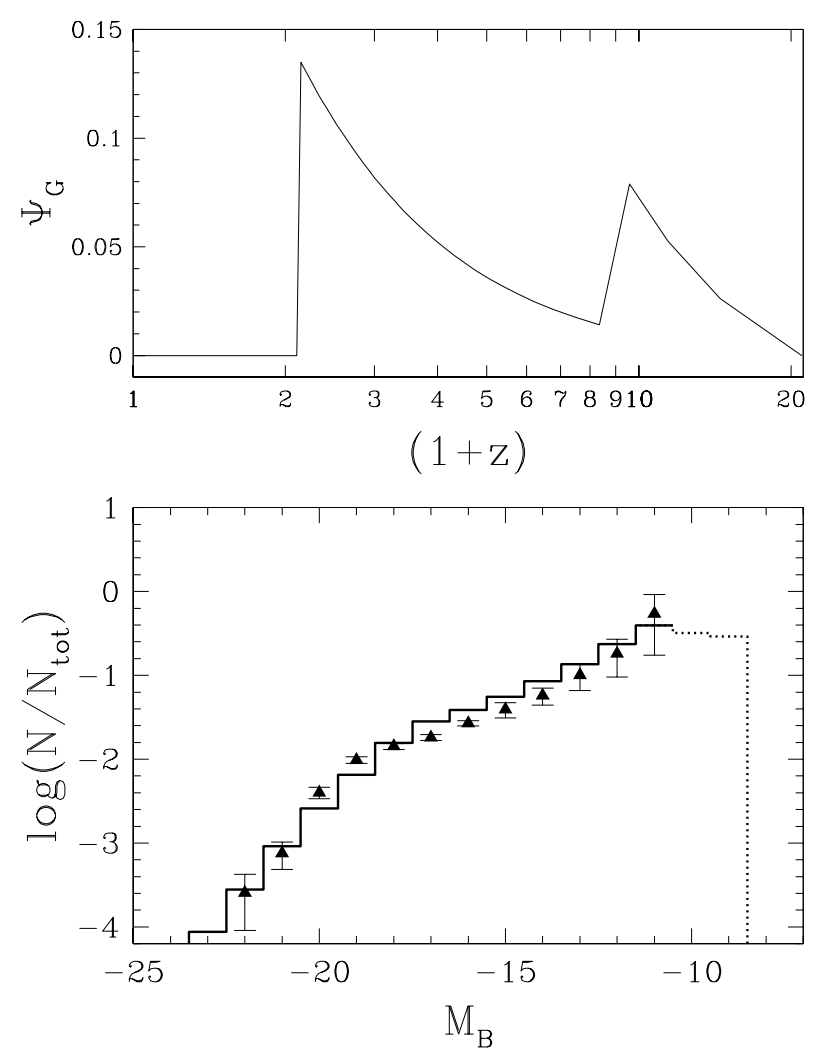

Fig. 8. An example of Galactic Formation History $\Psi_{\mathrm{G}}\left[\mathrm{Gyr}^{-1}\right]$ as a function of redshift and corresponding Luminosity Function (observational data by Trentham 1998). Model parameters are listed in Table 2.

detailed chemical network taking into account the different, finite stellar lifetimes at varying stellar mass (Sect. 3.1 and references therein).

- Moretti (2001) investigated a set of pre-chosen, arbitrary GFR laws, providing some indication as to what type of galaxy formation history is suited to reproduce the observed LF. An early burst of (dwarf) galaxy formation is needed to reproduce the steep faint end of the LF (see Fig. 7); this burst should be followed by a more gentle GFR increasing in time (i.e. at decreasing redshift) up to a certain epoch, dropping after that. Figure 8 shows such an example of suitable galaxy formation history $(\mathrm{GFH})$ and corresponding LF.

In the present model, we try to include this kind of behaviour self-consistently, by means of a "double infall" scheme.

- Further simplifying hypotheses regard the history and the morphology of the galaxies contributing to the chemical evolution of the cluster: in facts we have considered only elliptical non-interacting galaxies, since just early type galaxies seem to be responsible for the metal enrichment of the ICM (see Arnaud et al. 1992). Recent arguments about the baryon fraction in galaxies suggest however that early winds might be a general requirement for all objects (Silk 2003), so in this sense the distinction between elliptical or other galaxies might be of minor significance. We do not explicitly take into account the possibility of mergers nor of episodic star formation after the GW. 
- As to the cosmological parameters, which enter our model mostly through the time-redshift relation, we adopt a flat $\Lambda \mathrm{CDM}$ cosmology with $\Omega_{\mathrm{M}}=0.3, \Omega_{\Lambda}=0.7$ and $H_{0}=65 \mathrm{~km} \mathrm{~s}^{-1} \mathrm{Mpc}^{-1}$, corresponding to a present age of the Universe, or Hubble time, $t_{\mathrm{H}}=14.5 \mathrm{Gyr}$.

For the Press-Schechter Galactic IMF (see Sect. 5.2) we usually assume $n=1.5$ as the index of the power spectrum.

\subsection{Basic equations}

At any time the cluster is assumed to consist of the following components:

- The gas with primordial chemical composition and total mass $M_{\mathrm{g}, \mathrm{P}}(t)$, out of which galaxies are formed.

- The gas processed through galaxy formation and reexpelled by galaxies at the stage of galactic wind. Its total mass is $M_{\mathrm{g}, \mathrm{W}}(t)$. The wind-processed gas contains several chemical species (carbon, oxygen, iron, etc.). We will explicitely consider here only oxygen and iron, with total masses denoted by $M_{\mathrm{g}, \mathrm{O}}$ and $M_{\mathrm{g}, \mathrm{Fe}}$, respectively.

We remark here that "wind-processed gas" in our models indicates gas that has been processed through galaxies, not necessarily all of it through the stars in the galaxies. In fact, the GW is made both of metal enriched stellar ejecta and of pristine gas "energized" enough by stellar feed-back to leave the galaxy as wind, without having ever undergone actual star formation and stellar nucleosynthesis. This pristine gas in the GWs should not be confused with what we label as "primordial gas" $M_{\mathrm{g}, \mathrm{P}}$ in our cluster model, meant as gas that has never been involved in the process of galaxy formation.

- The galaxies, whose individual mass is the mass in stars and remnants left over after the GW stage - which occurs instantaneously as soon as the galaxy is formed, in the IRA. The total mass in galaxies is denoted by $M_{\mathrm{G}}(t)$.

- The total baryonic mass given by

$$
M_{\mathrm{b}}(t)=M_{\mathrm{g}, \mathrm{P}}(t)+M_{\mathrm{g}, \mathrm{W}}(t)+M_{\mathrm{G}}(t) .
$$

The Dark Matter component does not enter model equations as it does not intervene in the chemical evolution of the cluster.

As anticipated, for the primordial gas whence galaxies form we adopt a (double) infall scheme; this scheme is meant to describe not quite the accretion of baryons onto the cluster region, but the accretion onto the individual galaxies or, more precisely, the rate at which baryons become available (through cooling etc.) for galaxy formation. As pointed out by Pei \& Fall (1995), infall (or outflow) terms can be used even when dealing with large closed volumes, or with the whole Universe, as long as they represent gas exchange between the individual star forming objects and the surrounding medium. Owing to our double infall hypothesis, $M_{\mathrm{b}}$ increases in time by accretion of primordial gas following the equation

$$
\frac{\mathrm{d} M_{\mathrm{b}}}{\mathrm{d} t}=\left[\frac{\mathrm{d} M_{\mathrm{g}, \mathrm{P}}}{\mathrm{d} t}\right]_{\mathrm{inf}}=A t \mathrm{e}^{-\frac{t}{\tau_{1}}}+B\left(t-t_{0}\right) \mathrm{e}^{-\frac{t-t_{0}}{\tau_{2}}} .
$$

The first term represents a first, fast ( $\sim 0.5 \mathrm{Gyr})$ "infall episode" forming dwarf galaxies at high redshift; these objects are presumably responsible for reionization and/or reheating of the intergalactic medium, so that galaxy formation is temporarily halted. Later on, gas cools down again and galaxy formation progressively sets in again in a second, smoother and prolongued "infall phase" represented by the second term (active for $t>t_{0}$ ). This double infall scheme was chosen so as to yield a shape of the GFH suitable to reproduce the observed LF (from a preliminary investigation by Moretti 2001, see Fig. 8), as well as being reminiscent of current evidence and theories about reionization at high redshift and of the observed cosmic SFH of the Universe at lower redshift (the "Madau-plot").

The double infall scheme causes the baryonic mass of the cluster to increase up to a final value $M_{\mathrm{b}, \mathrm{T}}$ at the present time $t_{\mathrm{H}}$ (Hubble time, corresponding to $z=0$ ). We adopt this final total baryonic mass $M_{\mathrm{b}, \mathrm{T}}$ as the normalization mass to which all values are scaled:

$M_{\mathrm{b}}\left(t_{\mathrm{H}}\right)=M_{\mathrm{b}, \mathrm{T}}=1$.

Hence, in Eq. (3) we can tune the timescales $\tau_{1}$ and $\tau_{2}$ of the two infall episodes, as well as the amount of mass involved in the second infall phase (set by the parameter $B$ ), while the parameter $A$ is fixed by the normalization (4).

$A=\frac{1-B \tau_{2}\left[\tau_{2}-\left(\tau_{2}+t_{\mathrm{H}}-t_{0}\right) \mathrm{e}^{-\frac{t_{\mathrm{H}}-t_{0}}{\tau_{2}}}\right]}{\tau_{1}\left[\tau_{1}-\left(\tau_{1}+t_{\mathrm{H}}\right) \mathrm{e}^{-\frac{t_{\mathrm{H}}}{\tau_{1}}}\right]}$

With the adopted normalization (4), the masses of the different cluster components $\left(M_{\mathrm{g}, \mathrm{P}}, M_{\mathrm{g}, \mathrm{W}}\right.$ and $\left.M_{\mathrm{G}}\right)$ effectively become, as well as $M_{\mathrm{b}}$, mass fractions of the final baryonic mass $M_{\mathrm{b}, \mathrm{T}}$, and as such they are dimensionless quantities. The equations governing their temporal evolution are:

$$
\begin{aligned}
& \frac{\mathrm{d} M_{\mathrm{g}, \mathrm{P}}}{\mathrm{d} t}=-\Psi_{\mathrm{G}}(t)+\left[\frac{\mathrm{d} M_{\mathrm{g}, \mathrm{P}}}{\mathrm{d} t}\right]_{\mathrm{inf}} \\
& \frac{\mathrm{d} M_{\mathrm{g}, \mathrm{W}}}{\mathrm{d} t}=\Psi_{\mathrm{G}}(t) Y_{\mathrm{G}}(t) \\
& \frac{\mathrm{d} M_{\mathrm{G}}}{\mathrm{d} t}=\Psi_{\mathrm{G}}(t)\left[1-Y_{\mathrm{G}}(t)\right] .
\end{aligned}
$$

The equations governing the masses of oxygen and iron $M_{\mathrm{g}, \mathrm{O}}$ and $M_{\mathrm{g}, \mathrm{Fe}}$ expelled into the ICM by GWs are:

$$
\begin{aligned}
& \frac{\mathrm{d} M_{\mathrm{g}, \mathrm{O}}}{\mathrm{d} t}=\Psi_{\mathrm{G}}(t) Y_{\mathrm{G}, \mathrm{O}}(t) \\
& \frac{\mathrm{d} M_{\mathrm{g}, \mathrm{Fe}}}{\mathrm{d} t}=\Psi_{\mathrm{G}}(t) Y_{\mathrm{G}, \mathrm{Fe}}(t) .
\end{aligned}
$$

In the equations above, $\Psi_{\mathrm{G}}$ represents the Galaxy Formation Rate (GFR) - the analogue of the SFR in classical chemical models of the interstellar gas - while $Y_{\mathrm{G}}, Y_{\mathrm{G}, \mathrm{O}}$ and $Y_{\mathrm{G}, \mathrm{Fe}}$ are the "yields" per galactic generation of total gas, oxygen, and iron respectively; all these quantities will be specified in the following sections.

The total gas mass in the ICM is:

$M_{\mathrm{g}}=M_{\mathrm{g}, \mathrm{P}}+M_{\mathrm{g}, \mathrm{W}}$ 
and its oxygen and iron abundances are given by:

$X_{\mathrm{O}}=\frac{M_{\mathrm{g}, \mathrm{O}}}{M_{\mathrm{g}}} \quad X_{\mathrm{Fe}}=\frac{M_{\mathrm{g}, \mathrm{Fe}}}{M_{\mathrm{g}}}$.

Finally, the initial conditions for our set of equations are

$M_{\mathrm{g}, \mathrm{P}}(0)=0 \quad M_{\mathrm{g}, \mathrm{W}}(0)=0 \quad M_{\mathrm{G}}(0)=0$

together with

$M_{\mathrm{g}, \mathrm{O}}(0)=0 \quad M_{\mathrm{g}, \mathrm{Fe}}(0)=0$.

The set of equations is integrated with a 4th-order Runge-Kutta method, dividing the integration time-span [0 $t_{\mathrm{H}}$ ] into 1000 timesteps. The consistency of the solution is secured by the fact that at the final epoch $t=t_{\mathrm{H}}$ the sum of the different cluster components is:

$M_{\mathrm{g}}\left(t_{\mathrm{H}}\right)+M_{\mathrm{G}}\left(t_{\mathrm{H}}\right)=M_{\mathrm{b}, \mathrm{T}}=1$

within a small error (of the order of $10^{-3}$ ).

\subsection{Galactic Initial Mass Function}

The GIMF adopted here stems from the Press-Schechter (PS) mass function, originally devised to describe the collapse and formation of dark matter haloes from the primordial spectrum of perturbations. Following e.g. Lacey \& Cole (1994), the mass function of collapsed objects at redshift $z$ is:

$$
\begin{aligned}
\frac{\mathrm{d} f[z(t)]}{\mathrm{d} \ln M}= & \left(\frac{2}{\pi}\right)^{\frac{1}{2}}\left(\frac{n+3}{6}\right)\left(\frac{M}{M_{*}(z)}\right)^{\left(\frac{n+3}{6}\right)} \\
& \times \exp \left[-\frac{1}{2}\left(\frac{M}{M_{*}(z)}\right)^{\left(\frac{n+3}{3}\right)}\right]
\end{aligned}
$$

where $n$ is the index of the cosmological power spectrum, $z$ is the redshift corresponding to epoch $t$, and $M_{*}$ is the cut-off mass, or characteristic mass, at that redshift:

$$
M_{*}(z)=M_{\text {Nor }} \times(1+z)^{-\frac{6}{n+3}}, \quad M_{\text {Nor }}=M_{*}(z=0) .
$$

Equation (12) yields the fraction of mass in dark matter haloes with mass $M$ at redshift $z(t)$, per logarithmic mass interval. As discussed at the beginning of Sect. 5, for the purpose of the present study we assume that the same formula provides also the mass spectrum of the galaxies forming at redshift $z(t)$. For a fixed baryonic fraction in the Universe, typically $f_{\mathrm{b}}=0.1$, the ratio $M / M_{*}(z)$ in Eq. (12) can be considered both as the ratio between a dark halo mass and the corresponding "characteristic mass" at that redshift, and as the ratio between the corresponding baryonic masses. Namely, as the PS mass function is expressed solely in term of a mass ratio, it can be used both as a mass function of the dark haloes, and as a mass function of the corresponding baryonic components. In the latter case, $M_{*}(z)$ becomes the baryonic mass of the (proto)galaxy hosted by the "characteristic halo" at redshift $z$, or the typical mass-scale of (proto)galaxies at that redshift. In this context, $M_{\text {Nor }}$ is the mass of baryons contained in the typical dark halo mass collapsing at the present-day.

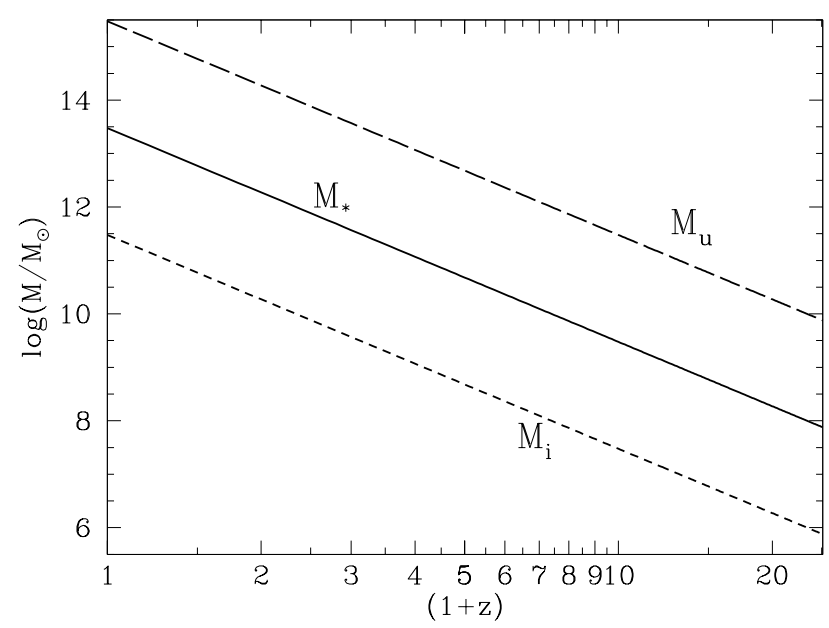

Fig. 9. Evolution of the characteristic mass $M_{*}$ (in this example, with $\left.M_{\mathrm{Nor}}=3 \times 10^{13} M_{\odot}\right)$ and of the minimum and maximum mass limits for the GIMF, $M_{\mathrm{i}}=M_{*} / 100$ and $M_{\mathrm{u}}=100 M_{*}$.

In our model the GIMF $\phi_{\mathrm{G}}$ is defined as the number of galaxies per mass interval, given by:

$\phi_{\mathrm{G}}(M, t)=\frac{1}{M^{2}} \times \frac{\mathrm{d} f[z(t)]}{\mathrm{d} \ln M}$

where the time dependence is due to $M_{*}(z(t))$ on the righthand side.

While Eqs. (12) and (14) define the shape of the GIMF, we need to define also the mass range $\left[M_{\mathrm{i}}, M_{\mathrm{u}}\right]$ within which galaxies can form at any redshift; in principle these mass limits can vary with redshift.

For the upper and lower mass limits we set, respectively:

$M_{\mathrm{u}}(z)=\gamma M_{*}(z) \quad M_{\mathrm{u}}(z)=\frac{M_{*}(z)}{\gamma}$

with $\gamma \simeq 100$; that is to say, at any given redshift galaxies form with masses within two orders of magnitude the characteristic mass at that redshift. Figure 9 shows an example of the evolution of $M_{*}, M_{\mathrm{i}}$ and $M_{\mathrm{u}}$ as a function of redshift (example with $M_{\text {Nor }}=3 \times 10^{13} M_{\odot}$ and $\left.\gamma=100\right)$.

The resulting galactic mass function is finally normalized in mass, at any redshift, according to:

$\int_{M_{\mathrm{i}}(t)}^{M_{\mathrm{u}}(t)} M \phi_{\mathrm{G}}(M, t) \mathrm{d} M=1$

so that $\phi_{\mathrm{G}}(M, t)$ properly becomes the distribution function of the masses of the galaxies formed at epoch $t$.

\subsection{Global galactic yields}

Once the mass function of galaxies and its mass limits are specified as a function of time (redshift), the global galactic yield of gas and metals of each galactic generation can be determined out of integration of the GW yields of individual galaxies over the mass function.

Our galactic models, discussed in Sect. 3.2, provide the mass of gas $E_{\mathrm{GW}}(M, t)$, iron $E_{\mathrm{GW}, \mathrm{Fe}}(M, t)$ and oxygen $E_{\mathrm{GW}, \mathrm{O}}(M, t)$, ejected in the $\mathrm{GW}$ by a galaxy of given initial 
mass $M$ and formation epoch $t$. The global quantities of gas, iron and oxygen instantaneously recycled and re-ejected into the ICM by an entire galaxy generation formed at epoch $t$ can be determined as:

$Y_{\mathrm{G}}(t)=\int_{M_{\mathrm{i}}(t)}^{M_{\mathrm{u}}(t)} E_{\mathrm{GW}}(M, t) \phi_{\mathrm{G}}(M, t) \mathrm{d} M$

$Y_{\mathrm{G}, \mathrm{Fe}}(t)=\int_{M_{\mathrm{i}}(t)}^{M_{\mathrm{u}}(t)} E_{\mathrm{GW}, \mathrm{Fe}}(M, t) \phi_{\mathrm{G}}(M, t) \mathrm{d} M$

$Y_{\mathrm{G}, \mathrm{O}}(t)=\int_{M_{\mathrm{i}}(t)}^{M_{\mathrm{u}}(t)} E_{\mathrm{GW}, \mathrm{O}}(M, t) \phi_{\mathrm{G}}(M, t) \mathrm{d} M$.

These are the quantities that enter Eqs. (6)-(9) and which must be evaluated at each timestep $t$ of the model, since all the quantities involved (GIMF, mass limits, GW ejecta) depend on the epoch $t$. The mass that remains locked in the galaxies ("galactic remnants", see Sect. 3.2) per galactic generation is:

$R_{\mathrm{G}}(t)=\int_{M_{\mathrm{i}}(t)}^{M_{\mathrm{u}}(t)} R_{\mathrm{gal}}(M, t) \phi_{\mathrm{G}}(M, t) \mathrm{d} M=1-Y_{\mathrm{G}}(t)$

entering Eq. (7).

\subsection{Galactic Formation Rate}

As to the Galactic Formation Rate, we adopt a simple Schmidt (1959) law

$\Psi_{\mathrm{G}}=v M_{\mathrm{g}, \mathrm{P}}$

where galaxies are assumed to form solely from the primordial gas component with a rate that is directly proportional to the available "fuel". With this law, the time evolution of the GFR is primarily set by the "infall history" of the primordial gas.

The Galaxy Formation History is supposed to start at epoch $z=20\left(\Psi_{\mathrm{G}}(t)=0\right.$ for $\left.z>20\right)$, and it is halted when the characteristic mass forming at that redshift, $M_{*}(z)$, becomes larger than that of the brightest observed galaxy in the LF. In our simple "hierarchical approach", namely, the formation of the brightest/largest galaxy corresponds to the lowest typical redshift at which galaxy formation is active.

\section{A fiducial model}

The main observational constraint to calibrate the Galaxy Formation History is the observed luminosity function. The LF by Trentham (1998, Fig. 7) shows the characteristic Schechterlike exponential cut-off at bright magnitudes $\left(M_{B}<-20\right)$, but it also steepens at the faint end $\left(M_{B}>-14\right)$. This large number of faint galaxies seems to require a prominent episode of formation of small galaxies at high redshift (Moretti 2001, and Fig. 8), a feature which inspired our present "double infall" prescription.

The LF of cluster galaxies in our models is calculated by assigning to each galaxy of "remnant" $R_{\text {gal }}\left(M, z_{\text {for }}\right)$ the relevant $M / L$ ratio for the age corresponding to its redshift of formation $z_{\text {for }}$ (see the discussion in Sect. 3.3).

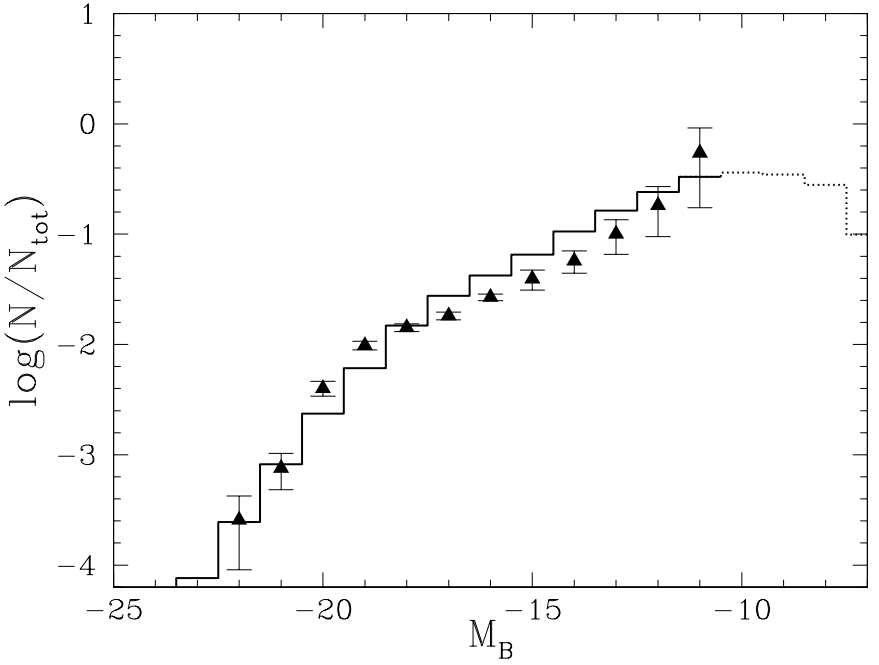

Fig. 10. Luminosity Function of cluster galaxies as predicted by our "fiducial" Model A; triangles are the observational data by Trentham (1998). Both the model LF and data are shown normalized to the same total (unit) number of objects in the observed range $\left(M_{B}\right.$ between -22 and $-11 \mathrm{mag})$.

As to the normalization of the total number of galaxies in the cluster, our models are calibrated also so that the final proportion of intra-cluster gas and galaxies respects the observational constraint of the ICMLR 37 (Sect. 4.2), within a $20 \%$.

Here we present and discuss in detail our "fiducial model", Model A whose parameters and final results are listed in Table 2. In this model we adopt as the characteristic mass for the GIMF (Sect. 5.2)

$M_{\text {Nor }}=M_{*}(z=0)=3 \times 10^{13} M_{\odot}$

which is of the order of the characteristic mass of galaxy clusters nowadays (Girardi et al. 1998) once a factor $f_{\mathrm{b}}=0.1$ is applied to scale the total mass to the mass of the sole baryonic component.

With the adopted parameters the final ICMLR $\sim 33$, the observed LF is reasonably well mimicked (Fig. 10) and the galactic GFH proceeds until $z \sim 1$ (Fig. 11); at lower redshifts, the typical galaxies forming would be more massive/bright than observed in the LF. The steep, faint end of the LF is reproduced by means of an early galaxy formation activity connected to the first infall episode (Fig. 11, top panel); this early activity is short $\left(\tau_{1} \sim 0.15 \mathrm{Gyr}\right)$ and can be identified with the formation of the first objects responsible for reionization. In fact, the second phase of galaxy formation (corresponding to the "second infall") slowly sets in starting from $t_{0}=0.8 \mathrm{Gyr}$, in broad agreement with recent observations suggesting that the reionization/reheating era took place at $z \gtrsim 6$ (Djorgovski et al. 2001; Becker et al. 2001). Hence, we can loosely associate the trough in the galaxy formation activity at the transition between the two "infall episodes" (Fig. 11) with the reheating of the intergalactic medium following the formation of the first galactic objects; the subsequent slow cooling down of the gas fuels the GFR at $z<6$. The GFH eventually halts so abruptly due to the simplified prescriptions in the model (Sect. 5.4); we remind 
Table 2. Parameters and results of the cluster models.

\begin{tabular}{|c|c|c|c|c|c|c|c|c|c|c|c|c|}
\hline Model & IMF & $M_{\text {Nor }}$ & $\tau_{1}$ & $\tau_{2}$ & $B$ & $v$ & $I M L R$ & $\frac{M_{\mathrm{gal}}}{L_{\mathrm{tot}}}$ & $\frac{M_{\mathrm{GW}}}{M_{\mathrm{gal}}}$ & $\frac{M_{\mathrm{ICM}}}{L_{\mathrm{tot}}}$ & $\frac{X_{\mathrm{Fe}, \mathrm{ICM}}}{X_{\mathrm{Fe}, \odot}}$ & {$[\mathrm{O} / \mathrm{Fe}]$} \\
\hline A & PNJ & $3 . e 13$ & 0.15 & 2.6 & 0.13 & 0.31 & 0.0058 & 5.6 & 1.56 & 32.8 & 0.096 & -0.061 \\
\hline AS & Salpeter & 3.e13 & 0.10 & 3.0 & 0.10 & 0.30 & 0.0013 & 7.1 & 0.72 & 38.8 & 0.019 & -0.085 \\
\hline M1 & PNJ & $5 . \mathrm{e} 12$ & 0.10 & 2.3 & 0.15 & 0.08 & 0.0042 & 4.4 & 1.53 & 29.9 & 0.076 & -0.064 \\
\hline A & PNJ & $3 . e 13$ & 0.15 & 2.6 & 0.13 & 0.31 & 0.0058 & 5.6 & 1.56 & 32.8 & 0.096 & -0.061 \\
\hline M2 & PNJ & 1.e14 & 0.10 & 0.9 & 0.80 & 0.25 & 0.0067 & 6.3 & 1.57 & 32.4 & 0.113 & -0.058 \\
\hline M3 & $\mathrm{PNJ}$ & 3.e14 & 0.01 & 0.8 & 0.50 & 0.29 & 0.0074 & 7.0 & 1.58 & 35.7 & 0.114 & -0.037 \\
\hline AfY & $\operatorname{PNJ}\left(z_{\text {for }}=15\right)$ & 3.e13 & 0.10 & 3.4 & 0.09 & 1.50 & 0.0184 & 8.2 & 2.26 & 40.6 & 0.247 & 0.163 \\
\hline $\mathrm{M} 2 \mathrm{fY}$ & $\operatorname{PNJ}\left(z_{\text {for }}=15\right)$ & 1.e14 & 0.10 & 1.6 & 0.35 & 1.25 & 0.0215 & 9.4 & 2.29 & 39.2 & 0.299 & 0.164 \\
\hline Fig. 8 & PNJ & 3.e13 & inpt & GFH & as in $\mathrm{F}$ & g. 8 & 0.0061 & 5.8 & 1.56 & 30.7 & 0.108 & -0.060 \\
\hline
\end{tabular}
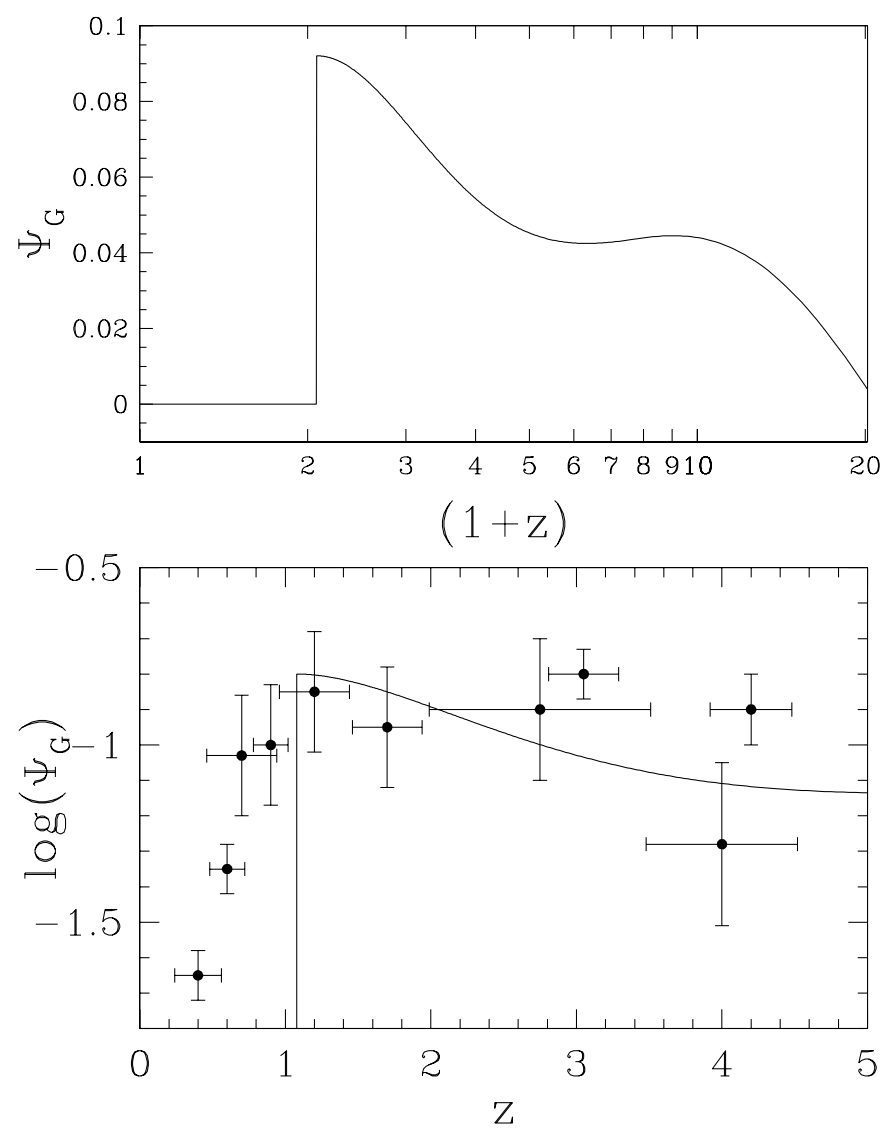

Fig. 11. Top panel: GFH of our Model A as a function of redshift; $\Psi_{\mathrm{G}}$ is expressed in mass fraction of the cluster per Gyr. Bottom panel: comparison of the shape of our GFH with the "Madau plot" (data from Steidel et al. 1999); the theoretical curve $\Psi_{\mathrm{G}}$ is arbitrarily normalized so that its maximum value reaches $\log \left(\Psi_{\mathrm{G}}\right)=-0.8$, similar to the maximum in the data.

also that only early-type galaxies (E/S0) are considered in our cluster model, while in the Madau plot for field galaxies, most of the SF at $z<1$ is due to spirals.

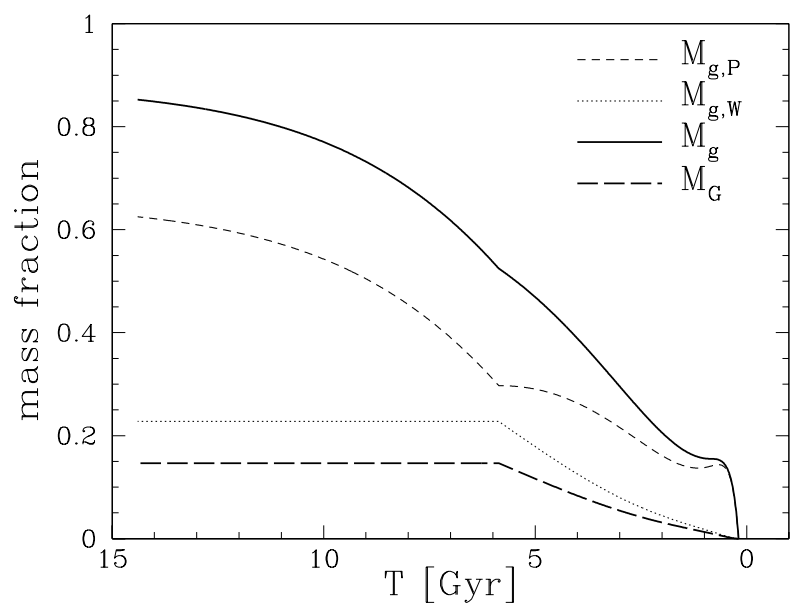

Fig. 12. Time evolution of the cluster components for Model A.

In Fig. 10, the dotted part of the histogram represent the predicted extension of the LF to the range of galaxies fainter than the observational limit. Although these small, faint galaxies dominate in number, their contribution in terms of luminosity or stellar mass is a negligible fraction of the total (see also Thomas 1999); in the Local Group as well, the numerous dwarf galaxies play a negligible role in the global mass and luminosity budget. In clusters, these faint objects might also have been disrupted and be nowadays dispersed as a diffuse intra-cluster stellar component (Gregg \& West 1998; Ferguson et al. 1998; Arnaboldi et al. 2002, and references therein).

Figure 12 shows the evolution of the mass fractions in the various cluster components: the mass in primordial gas, in wind-processed gas and in galaxies. The "double infall" pattern can be recognized in the evolution of the primordial gas component; within the first Gyr, the early infall phase produces a minor peak - first the gas mass increases due to infall, then it tends to decrease, being consumed by galaxy formation; later on, again the increase due to infall competes with the consumption by galaxy formation, until the latter halts at $z=1$. At the end of the evolution, wind-processed gas is $\sim 1.5$ times the mass in galaxies, in agreement with qualitative expectations 


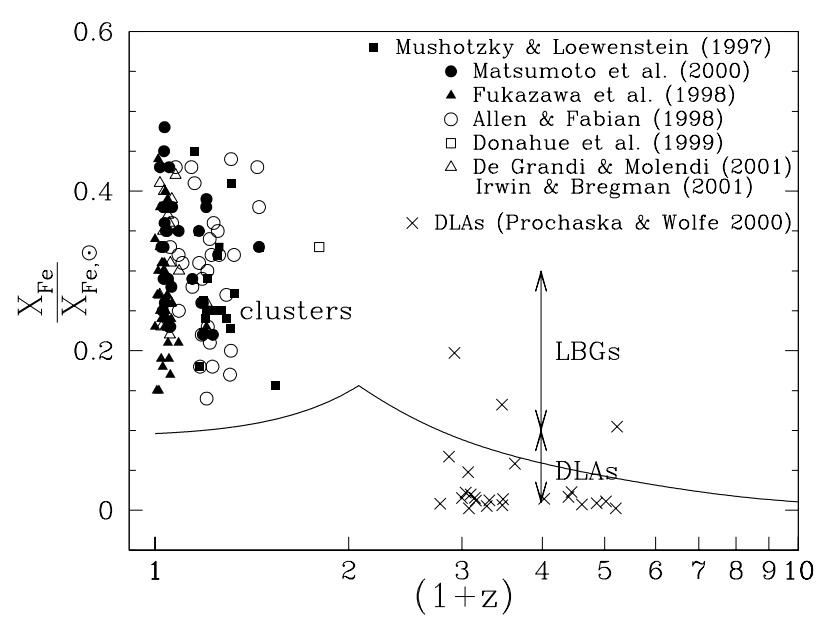

Fig. 13. Evolution of the iron abundance in the ICM. At high redshift $(z>1.5)$ metallicities for Damped Lyman $\alpha$ systems and Lyman Break Galaxies are shown for the sake of qualitative comparison (metallicity ranges for LBGs and DLAs at $z=3$ are from Pettini 2000).

from individual galaxies with the PNJ IMF (Sect. 3.2). The total mass in gas is about 6 times the mass in galaxies; about $70 \%$ of the ICM is primordial gas never involved in the galaxy formation process.

Figure 13 shows the time evolution of metallicity (iron abundance) in the ICM; it peaks at $z \sim 1$, when the most massive galaxies are formed and release their metal production, then it decreases because primordial gas is still being accreted onto the cluster, following our infall prescription, even after galaxy formation and metal production are over. The final metallicity is low by a factor of 3 with respect to observations, in agreement with the low overall IMLR $(\sim 0.006$, Table 2$)$. This result is easily understood since most galaxies (in terms of mass involved) form at redshifts $z \leq 5$, with a low corresponding IMLR (see the discussion in Sect. 4 and Table 2).

The predicted $[\mathrm{O} / \mathrm{Fe}]$ ratio is roughly solar (Table 2 ). If we were to compensate for the low iron production by increasing the rate and iron contribution of SN Ia by the required factor of 3 , the resulting $[\mathrm{O} / \mathrm{Fe}]$ ratio would decrease by $0.5 \mathrm{dex}$, and become too low with respect to observations, which indicate ratios between solar and supersolar for the bulk of the ICM (Finoguenov et al. 2000).

\subsection{Comparison to the Salpeter case}

For the sake of comparison, we calculate an analogous model (Model AS, with parameters listed in Table 2) adopting GW ejecta and "galactic remnants" as computed with the Salpeter IMF. Figure 14 is the analogue of Figs. 10 to 13 for Model AS. The LF is reproduced with a GFH qualitatively similar to that of Model A, but both the metal abundances in the ICM and the IMLR (Table 2) are far from observational data. Besides, in this case the mass of wind-ejected gas is comparable to, or lower $(70 \%)$ than, the mass in galaxies and constitutes a minor fraction $(13 \%)$ of the total final ICM.

Namely, with a Salpeter IMF galaxies produce by far too little metals per mass stored in galaxies. With the PNJ IMF things visibly improve (Fig. 13), still the expected metallicity is $\sim 3$ times lower than observed.

\section{Varying the Galaxy Formation History}

In this section we explore alternative models with "slower" or "faster" GFHs, by considering different characteristic masses $M_{\text {Nor }}$ for the GIMF (Sect. 5.2). Qualitatively, increasing $M_{\text {Nor }}$, the galaxies forming at a given redshift are intrinsically more massive and luminous, so that to reproduce the observed LF the optimal GFH is "anticipated", or skewed to higher $z$. Thus, $M_{\text {Nor }}$ is linked to the peak (and halt) of the corresponding GFH. Similar effects could be obtained also by changing the spectral index $n$, for a shallower power-spectrum also induces the formation of intrinsically more massive galaxies at a given redshift, henceforth "anticipating" the required GFH. We explored models with $n$ values different from the 1.5 adopted here, but we do not report them here for the sake of brevity, as conclusions would be similar to those with varying $M_{\text {Nor }}$.

Models M1, M2 and M3 are analogous to Model A, but for different values of $M_{\text {Nor }}$; in each model, the "secondary parameters" governing the GFH $\left(B, \tau_{1}, \tau_{2}, v\right)$ are optimized so as to reproduce the observed LF and ICMLR. Model M1 has $M_{\text {Nor }}=5 \times 10^{12} M_{\odot}$, comparable to the characteristic mass at $z=0$ in the field, which in current cosmologies is of the order of a few $10^{13} M_{\odot}$ (total mass, dark matter included). Models M2 and M3 have a value of $M_{\text {Nor }}$ larger than in Model A; their $M_{\text {Nor }}$ corresponds to the typical baryonic content of rich clusters, with total mass of the order of $10^{15} M_{\odot}$.

As anticipated, at increasing $M_{\text {Nor }}$ (from M1 to M3), the GFH is skewed to higher $z$ (Fig. 15, d-panels); also the bulk of the metal production is correspondingly anticipated. The main consequence is that the global IMLR increases with $M_{\text {Nor }}$ (Table 2), since a given $L_{B}$ bin in the LF now corresponds to older, dimmer and more massive galaxies, which eject both more gas and more metals into the ICM, and have a higher characteristic $I M L R_{\mathrm{GW}}$ (Sect. 3.2 and 3.3). Nevertheless, even in model M3 corresponding to the largest $M_{\mathrm{Nor}}$, the predicted $I M L R$ is still low by a factor of 2 with respect to observations. This is due to the fact that in all models most galaxies in terms of mass involved form anyways at $z \leq 5$, with a low characteristic $I M L R$, as was the case for model A. Correspondingly, the predicted metallicities are too low.

Hence, considering different GFHs - in particular, "anticipated" GFHs with respect to model A, as in models M2 and M3 - improves the situation with metal production, but only slightly. The conclusion in the previous section still holds, that if an IMF varying with redshift performs much better than the Salpeter models, the metal production provided by the PNJ models is still low.

\section{Choosing the IMF "ad hoc"}

In the previous sections we showed that a varying IMF like the PNJ IMF, skewed toward more massive stars (or more precisely, having a lower locked-up fraction) at higher redshifts, yields much better results for the predicted ICM metallicity, 

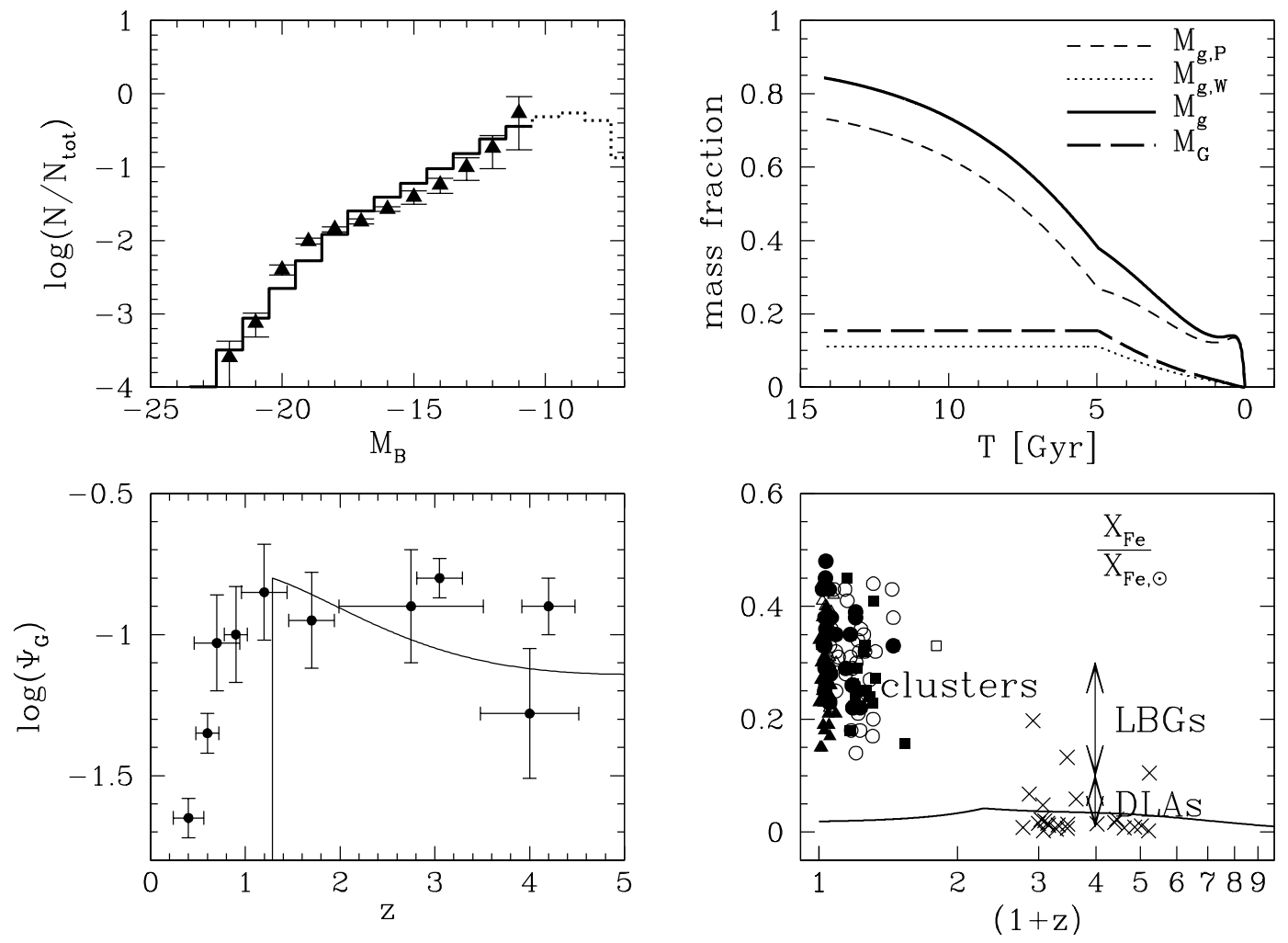

Fig. 14. Same as Figs. 10-13, but for Model AS based on the Salpeter IMF.

than the standard Salpeter IMF. Nevertheless, a still larger metal production seems to be required, by a factor of 2-3, than obtained with our PNJ models. In these models, aside from the variation with galaxy mass due to density effects, the peak mass of the IMF mainly varies with redshift because of the CMB temperature (Sect. 3.1); apparently, a larger variation is required to reach the required metal production. Interestingly, this is in line with other recent results: both Hernandez \& Ferrara (2001), from the number of metal-poor stars in the halo of the Milky Way, and Finoguenov et al. (2003), from arguments related to the different yield in groups and clusters, suggest a typical peak-mass of the IMF increasing with redshift faster than what expected from the pure Jeans effect of the CMB temperature.

We consider in this section some cluster models assuming an IMF that is more efficient in terms of gas and metal ejection in the galactic wind. We achieve this by "fixing" the galaxy models to be the PNJ models with $z_{\text {for }}=15$ : the GFH in the cluster still extends in time down to $z \sim 1$ (or lower), but now we assume that galaxies at any redshifts form with the properties (galactic yields and remnants) of the PNJ models with $z_{\text {for }}=15$. Namely, the integrals 15 through 18 in Sect. 5.3 are now computed always from the models with $z_{\text {for }}=15$, rather than with a $z_{\text {for }}$ running with the actual epoch $z$ of cluster evolution. This is a simple artifact to mantain an IMF "more efficient" than the CMB-regulated PNJ IMF. Luminosities are of course computed for the actual age $t(z)$ of the galaxies. The choice of the models with $z_{\text {for }}=15$ is ad hoc to obtain the correct final IMLR and metallicity (see Sect. 4 and Fig. 16).
The IMLR resulting from the "hierarchical" GFH is somewhat lower than the monolithic case with the same $z_{\text {for }}=15$ because, with galaxy formation extending down to redshift 1-2, galaxies are on average younger and more luminous than in the monolithic case, lowering the typical $I M L R_{\mathrm{GW}}$ for a given mass of ejected iron.

In Fig. 16 we show the cluster models AfY and M2fY, analogous to models A and M2 (i.e. with the same $M_{\text {Nor }}=$ $3 \times 10^{13}$ and $10^{14}$, respectively) but calculated with "fixed galactic yields" as described above; parameters and results are listed in Table 2 . In both cases the final metallicity and IMLR are in good agreement with observations, the mass in ejected wind is $\sim 2.3$ times the mass in galaxies and constitutes roughly half of the whole ICM gas. Notice also that, for these models reproducing the correct final ICM metallicity, the predicted metallicity at redshift 3-5 falls in the range of Lyman Break Galaxies, which are in fact considered to trace the high redshift counterpart of present-day massive galaxies in high density regions of the Universe, such as clusters.

In model AfY, the GFH peaks at $z \sim 1$ (similarly to the Madau plot), and correspondingly a noticeable peak in metallicity is predicted at the same redshift, for no further enrichment takes place for the primordial gas infalling onto the cluster later on. This fast metallicity evolution is not really observed - although very few data points are available for redshifts $z>0.6$, where most of the evolution takes place.

A milder behaviour, somewhat more compatible with data, is found for model M2fY, having a characteristic $z=0$ mass $M_{\text {Nor }}$ of the order of the baryonic mass in rich clusters. 

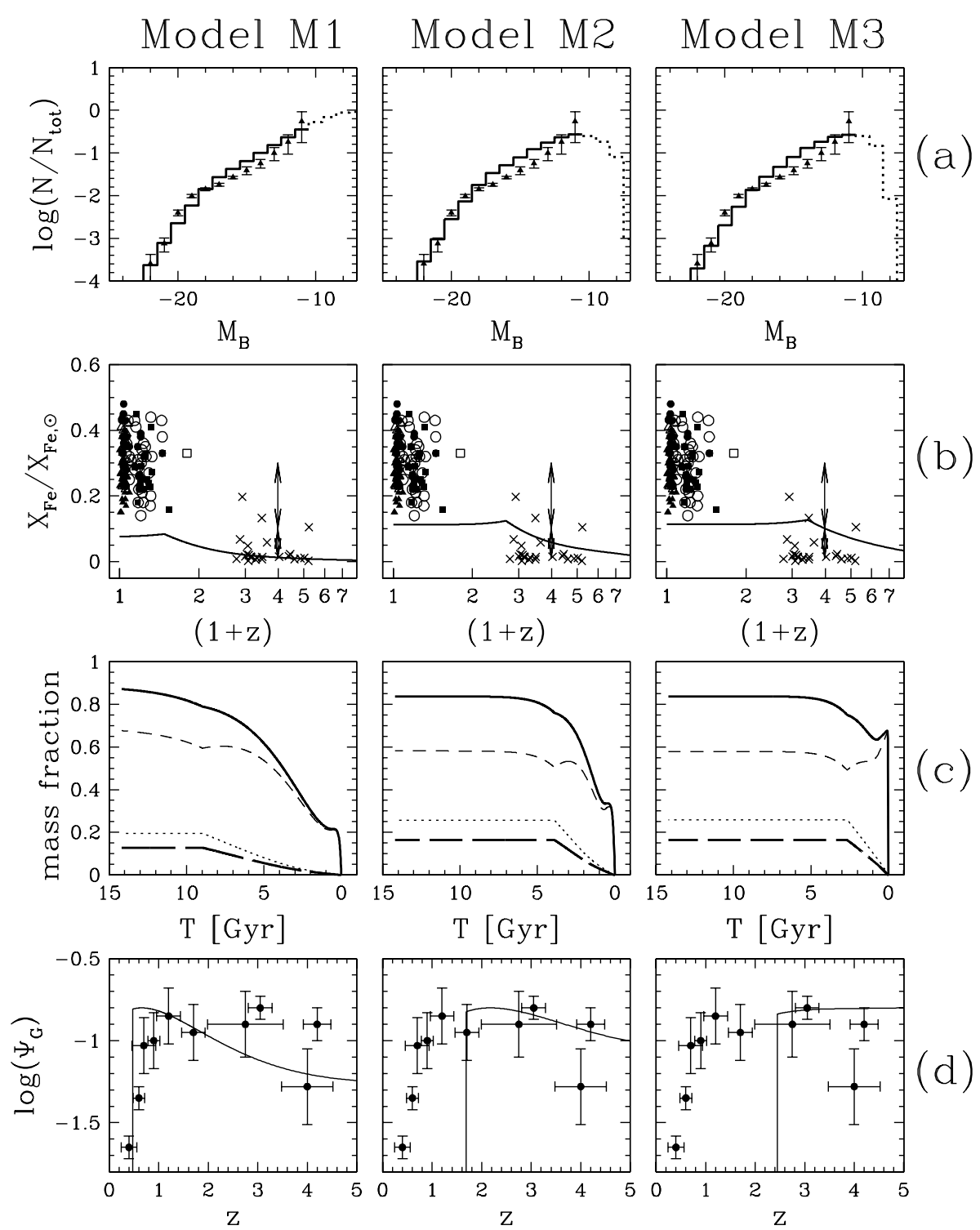

Fig. 15. Results for Models M1, M2 and M3 which differ from our fiducial Model A in the characteristic mass $M_{\text {Nor }}$, increasing from M1 to M3 (Table 2). a) Luminosity Function; b) evolution of the iron abundance; c) evolution of the cluster components; d) Galactic Formation History. Lines, symbols and data as in Figs. 10 to 13.

For this model, the GFH peaks at $z \sim 2$ and halts at $z \sim 1.5$, the metallicity peaks at $z \sim 1.5$ while evolving rather smoothly out to $z \sim 1$. We consider model M2fY as our best model.

\section{Abundance ratios in the ICM}

As mentioned in the introduction, the typical $[\alpha / \mathrm{Fe}]$ abundance ratios in the ICM are not firmly established from observations. With the first ASCA data, it was debated if the ICM is typically $\alpha$-enriched or if its abundances are consistent with solar ratios (Mushotzky et al. 1996; Ishimaru \& Arimoto 1997). Recent studies have revealed that abundance ratios may change in different regions of the cluster, from roughly solar in the central regions to $\alpha$-enriched in the outer regions (Finoguenov et al. 2000). This might suggest competing, or rather overlapping, extraction mechanisms of metals from galaxies: SN II ejecta would dominate in GWs, while additional SN Ia products would be extracted from the galaxies due to ram-pressure stripping, more effective in the central regions (Finoguenov et al. 2000).

Our model at present does not deal with gradients of abundance or of abundance ratios in the ICM, however it provides the global oxygen mass produced by galaxies and ejected into the ICM by GWs, so that we can estimate the typical average $[\mathrm{O} / \mathrm{Fe}]$ ratio in the ICM, with oxygen being the best tracer of $\alpha$-elements. In Fig. 17 we show the evolution of the [O/Fe] ratio in the ICM as predicted from our reference Model A; $[\mathrm{O} / \mathrm{Fe}]$ predictions for models $\mathrm{M} 1-\mathrm{M} 2-\mathrm{M} 3$ are very similar so these models are not shown in the plot. $[\mathrm{O} / \mathrm{Fe}]$ is supersolar at very high redshifts (where the PNJ IMF favours more massive stars and hence SN II), decreasing down to marginally subsolar 

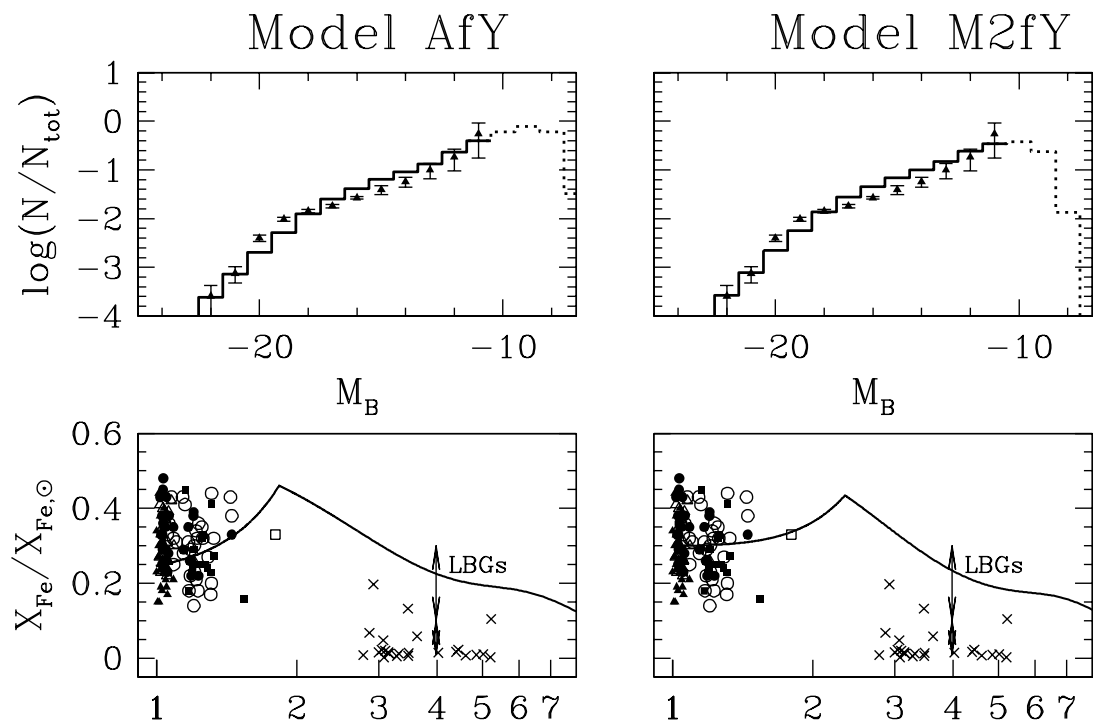

(b)
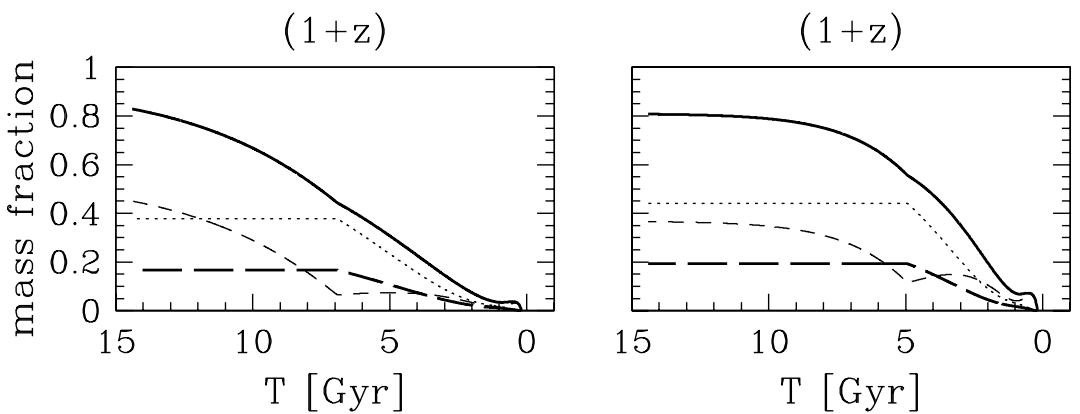

(c)
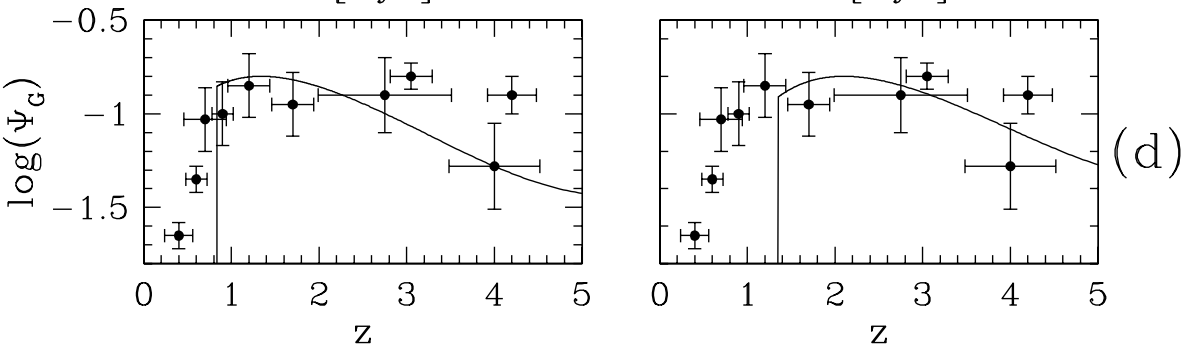

Fig. 16. Results for Models AfYz15 and M2fYz15, analogous to models A and M2 but with fixed galactic yields corresponding to the PNJ models with $z=15$. Panels and symbols as in Fig. 15.

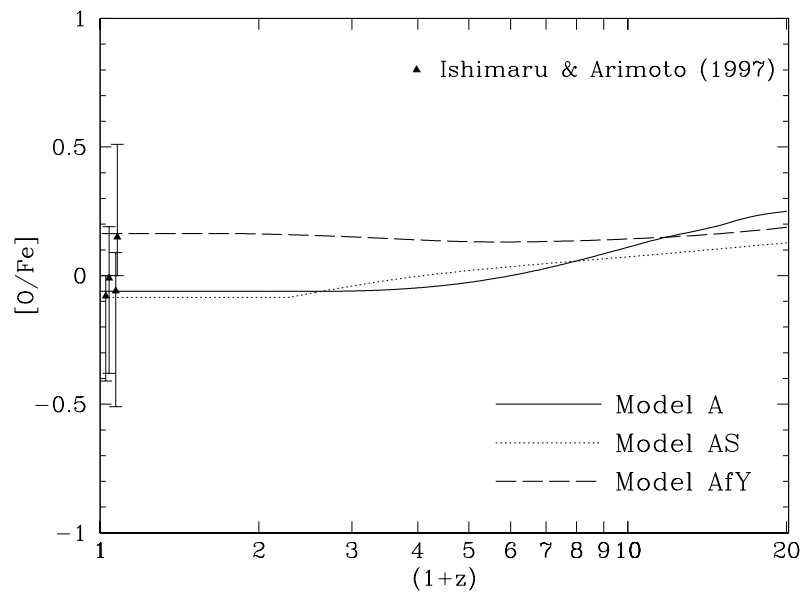

Fig. 17. Evolution of the $[\mathrm{O} / \mathrm{Fe}]$ abundance ratio as a function of redshift for our Models A, AS and AfY, compared to observations for low redshift clusters. values. Model AS with the Salpeter IMF is also shown for comparison; the final $[\mathrm{O} / \mathrm{Fe}]$ value in very close to that of Model $\mathrm{A}$.

Also shown is model AfY with the "fixed yields" PNJ models (model M2fY is basically indistinguishable from AfY in this plot and is not shown); the predicted final $[\mathrm{O} / \mathrm{Fe}]$ is slightly supersolar (around $+0.2 \mathrm{dex}$ ). Due to the "fixed yields" assumption in this model, $[\mathrm{O} / \mathrm{Fe}]$ is roughly constant throughout the evolution and its value is characteristic of the PNJ models with $z_{\text {for }}=15$ (see Sect. 4).

Given all the above mentioned caveats about the complexity and uncertainties of the empirical evidence, we consider all these final values to be in broad agreement with observations. Notice however that all models predict a very slow evolution (if any) in the $[\mathrm{O} / \mathrm{Fe}]$ ratio, so that no sistematic trend is expected to be seen in for this observable until $z>3$ at the earliest. 


\section{Summary and conclusions}

Galactic winds from elliptical galaxies are the most likely source of the chemical enrichment in the ICM. In this scenario, various studies in literature suggest that a non-standard IMF must be invoked for elliptical galaxies, if we are to account for the metallicity of the ICM (Sect. 2). Chiosi et al. (1998), Chiosi (2000b) calculated models of elliptical galaxies adopting the variable IMF by PNJ, whose behaviour in time and space is sensitive to the physical conditions of the star-forming gas; in particular, the low-mass cut behaves as a sort of Jeans mass (Sect. 3). This IMF naturally predicts a lower locked-up fraction in the early galactic stages, especially for massive ellipticals and/or for high redshifts of formation the latter feature being related to the increasing temperature of the CMB. These galactic models successfully reproduce a variety of observational properties of ellipticals (Chiosi et al. 1998). In Sect. 3.2 we show how models calculated with the PNJ IMF predict galaxies to eject both more metals and more gas than "standard" models based on the Salpeter IMF. We also show that, for the more massive objects that play the main role in the mass and metal enrichment budget of the cluster, the characteristic IMLR of individual galaxies is much higher for models with the PNJ IMF than with the Salpeter IMF (Sect. 3.3).

To assess the effect of these new galactic models on the ICM, first we assumed all galaxies to be coeval and we integrated the corresponding ejecta over the observed luminosity function, as in the standard "monolithic approach" (Sect. 4). The Salpeter models fail in reproducing the observed IMLR in clusters by an order of magnitude, while better results are obtained with the the PNJ IMF, especially with the models corresponding to a redshifts of formation $z_{\text {for }} \sim 13$. We also discuss the evidence of dilution of the galactic wind ejecta with primordial gas, introducing the concept of the intra-cluster-mass-tolight ratio $(I C M L R)$; for the typical luminosities and $M / L$ ratios of our model galaxies, a dilution of a factor 2-3 for the PNJ models (a factor of 6 for the Salpeter models) is necessary to recover the typical amount of gas vs. galaxies observed in rich clusters.

Alternatively to the standard monolithic approach, we considered a simple hierarchical picture in which galaxy formation extends in time, with a characteristic galaxy mass increasing at decreasing redshift, as from Press-Schechter theory. We developed a toy-model following the chemical evolution of the ICM in connection with the galaxy formation history of cluster galaxies (Sect. 5). The GFH is calibrated so as to reproduce the observed present-day galactic LF in clusters, and the observed amount of ICM gas (in terms of ICMLR). This seems to require two phases of galaxy formation: an early phase forming dwarf galaxies populating the steep, faint tail of the LF, followed by a more gentle GF activity peaking at $z \sim 1-2$. This scenario is in broad agreement with (a) present theories and observational evidence about reionization and reheating of the Universe at $z \gtrsim 6$, and (b) the observed trend of cosmic star formation history - although in our cluster models the GFH is "anticipated" and halts earlier with respect to the field-based Madau-plot, since we are considering early-type galaxies. We mimick this "bimodal" galaxy formation history by means of a double infall prescription in our chemical model for clusters.

A satisfactory match with the observed LF can be obtained both using galactic models with the PNJ IMF and with the Salpeter IMF. However, models with the PNJ IMF, besides being favoured on the base of their photometric properties (Chiosi et al. 1998), provide much improved predictions about the metal enrichment of the ICM; Salpeter-based models fail in this respect (Sect. 6). Still, the PNJ models are short of metal production by a factor of 2-3, and the conclusion does not depend much on the details of the GFH (Sect. 7). In fact, with such extended GFHs the bulk of galaxies (in terms of mass involved) form at redshifts $z<5$, where the PNJ models do have a typical IMLR which is a factor of 2-3 too low (Sect. 4).

The discrepancy cannot be cured simply by increasing the (quite uncertain) rate of SN Ia in the models, since the resulting $[\mathrm{O} / \mathrm{Fe}]$ ratio would correspondingly decrease by $0.3-0.5 \mathrm{dex}$ at odds with observations. Our models presently give the correct relative contribution of SN Ia and SN II as demonstrated by the predicted $[\mathrm{O} / \mathrm{Fe}]$ ratio in the ICM (solar or slightly supersolar, Sect. 9) and strong variations of the SN Ia rate are not allowed within the constraint of the observed relative abundances.

The implication is that an IMF with a more extreme behaviour than assumed in our galactic models is needed. Variations of the stellar Jeans mass with redshift induced by the CMB temperature appear to be too mild to produce the observed metal content in clusters, in agreement with other recent results (Hernandez \& Ferrara 2001; Finoguenov et al. 2003). In fact, from the monolithic approach an IMF is required, behaving like that in the PNJ models with $z_{\text {for }} \sim 13-15$.

We thus computed some cluster models with the IMF chosen "ad hoc" to induce a metal ejection large enough to enrich the ICM to the observed abundance level (Sect. 8). Our best model is M2fY (Fig. 16), with a GFH peaking at $z \sim 2$ and halted by $z \sim 1.5$. This models predicts a peak in the metallicity of the ICM around $z \sim 1.5$, decreasing at higher redshifts; at $z=3-4$ the typical metallicity corresponds to observed Lyman-break galaxies. In this model, the wind-ejected gas is more than twice the mass in galaxies, and constitutes roughly half of the ICM mass.

In general, when galactic models with the PNJ IMF are adopted, the mass globally ejected in GWs exceeds the mass stored in stars by a factor of 1.5-2 - arguments in favour of large outflows of baryons from the main body of galaxies have been recently advanced also by Silk (2003). Although this is not enough to account for the total mass in the ICM, it is nevertheless a non-negligible fraction of the intra-cluster gas, especially for less rich clusters. This suggestion is reinforced by recent results obtained with dynamical models of galaxy formation (Carraro et al. 2001; Chiosi \& Carraro 2002), showing that the process of galaxy formation is so unefficient that roughly $75 \%$ of the gas initially available is "wasted" and expelled, while only a $25 \%$ remains locked in the final galaxy. These dynamical models, even adopting a constant IMF, suggest a typical ratio of 1:3 between the matter stored in galaxies and that re-ejected in the inter-galactic, or intra-cluster, medium. It is suggestive to speculate that 3:1 or so could set a minimal value for the gas-to-galaxy mass ratio in clusters; 
this value could then be typical of poor clusters, whose ICM would be dominated by gas of galactic origin. This is also in line with the observational evidence that the energetics of the ICM in the less rich and luminous clusters are dominated by non-gravitational effects, most likely related to the energy feed-back from cluster galaxies.

Acknowledgements. We would like to thank our referee, Prof. A. Renzini, for very helpful critical remarks.

We benefitted also from discussions with S. Andreon, A. Finoguenov, M. Götz, E. Pignatelli and J. Sommer-Larsen. LP acknowledges kind hospitality from the Astronomy Department in Padova and from the Observatory of Helsinki upon various visits.

This study was financed by the Italian MIUR and University of Padova under the special contract "Formation and evolution of Elliptical Galaxies" and by the Danmarks Grundforskningsfond (through its support to TAC).

\section{References}

Abel, T., Bryan, G. L., \& Norman, M. L. 2002, Science, 295, 93

Andreon, S., \& Cuillandre, J. C. 2002, ApJ, 569, 144

Andreon, S., \& Pelló, R. 2000, A\&A, 353, 479

Arimoto, N., Matsushita, K., Yshimaru, Y., Osaki, T., \& Renzini, A. 1997, ApJ, 477, 128

Arimoto, N., \& Yoshii, Y. 1987, A\&A, 173, 23

Arnaboldi, M., Aguerri, J. A. L., Napolitano, N. R., et al. 2002, AJ, 123,760

Arnaud, M., Rothenflug, R., Boulade, O., Vigroux, L., \& Vangioni-Flam, E. 1992, A\&A, 254, 49

Balogh, M. L., Pearce, F. R., Bower, R. G., \& Kay, S. T. 2001, MNRAS, 326, 1228

Becker, R. H., Fan, X., White, R. L., et al. 2001, AJ, 122, 2850

Bica, E., Bonatto, C., Pastoriza, M. G., \& Alloin, D. 1996, A\&A, 313, 405

Bressan, A., Chiosi, C., \& Fagotto, F. 1994, ApJS, 94, 63

Briceño, C., Luhman, K., Hartmann, L., Stauffer, J., \& Kirkpatrick, D. 2002, ApJ, 580, 317

Bromm, V., Coppi, P. S., \& Larson, R. B. 2002, ApJ, 564, 23

Bryan, G. L. 2000, ApJ, 544, L1

Buote, D. A. 2003, in IGM/Galaxy Connection - The Distribution of Baryons at $z=0$, ed. J. L. Rosenberg, \& M. E. Putman, ASSL, 281, 87

Carraro, G., Chiosi, C., Girardi, L., \& Lia, C. 2001, MNRAS, 327, 69 Chabrier, G. 2003, PASP, 115, 763

Chiosi, C. 2000a, in ed. A. Weiss, T. Abel, \& V. Hill, The First Stars (Springer), 95

Chiosi, C. 2000b, A\&A, 364, 423

Chiosi, C., Bressan, A., Portinari, L., \& Tantalo, R. 1998, A\&A, 339, 355

Chiosi, C., \& Carraro, G. 2002, MNRAS, 335, 335

Ciotti, L., D’Ercole, A., Pellegrini, S., \& Renzini, A. 1991, ApJ, 376, 380

Cirimele, G., Nesci, R., \& Trevese, D. 1997, ApJ, 475, 11

David, L. P., Arnaud, K. A., Forman, W., \& Jones, C. 1990, ApJ, 356, 32

David, L. P., Forman, W., \& Jones, C. 1991a, ApJ, 380, 39

David, L. P., Forman, W., \& Jones, C. 1991b, ApJ, 369, 121

De Grandi, S., \& Molendi, S. 2001, ApJ, 551, 153
De Propris, R., Stanford, S. A., Eisenhardt, P. R., Dickinson, M., \& Elston, R. 1999, ApJ, 118, 719

De Young, D. S. 1978, ApJ, 223, 47

Djorgovski, S. G., Castro, S., Stern, D., \& Mahabal, A. A. 2001, ApJ, 560, L5

Driver, S. P., Couch, W. J., \& Phillips, S. 1998, MNRAS, 301, 369

Elbaz, D., Arnaud, M., \& Vangioni-Flam, E. 1995, A\&A, 303, 345

Ettori, S., Fabian, A. C., Allen, S. W., \& Johnstone, R. M. 2002, MNRAS, 331, 635

Ferguson, H. C., Dickinson, M., \& Papovich, C. 2002, ApJ, 569, L65

Ferguson, H. C., Tanvir, N. R., \& von Hippel, T. 1998, Nature, 391, 461

Finoguenov, A., Arnaud, M., \& David, L. P. 2001, ApJ, 555, 191

Finoguenov, A., Burkert, A., \& Böhringer, H. 2003, ApJ, in press [astro-ph/0305190]

Finoguenov, A., David, L. P., \& Ponman, T. J. 2000, ApJ, 544, 188

Fukazawa, Y., Makishima, K., Tamura, T., et al. 1998, PASJ, 50, 187

Fukazawa, Y., Makishima, K., Tamura, T., et al. 2000, MNRAS, 313, 21

Gibson, B. K., \& Matteucci, F. 1997a, MNRAS, 291, L8

Gibson, B. K., \& Matteucci, F. 1997b, ApJ, 475, 47

Girardi, M., Borgani, S., Giuricin, G., Mardirossian, F., \& Mezzetti, M. 1998, ApJ, 506, 45

Gregg, M. D., \& West, M. J. 1998, Nature, 396, 549

Greggio, L., \& Renzini, A. 1983, A\&A, 118, 217

Hernandez, X., \& Ferrara, A. 2001, MNRAS, 324, 484

Hinnes, A., \& Biermann, P. 1980, A\&A, 86, 11

Irwin, J. A., \& Bregman, J. N. 2001, ApJ, 546, 150

Ishimaru, Y., \& Arimoto, N. 1997, PASJ, 49, 1

Johnstone, R. M., Allen, S. W., Fabian, A. C., \& Sanders, J. S. 2002, MNRAS, 336, 299

Kaastra, J. S., Ferrigno, C., Tamura, T., et al. 2001, A\&A, 365, L99

Lacey, C., \& Cole, S. 1994, MNRAS, 271, 676

Larson, R. B. 1974, MNRAS, 169, 229

Larson, R. B. 1986, MNRAS, 218, 409

Larson, R. B. 1998, MNRAS, 301, 569

Larson, R. B., \& Dinerstein, H. L. 1975, PASP, 87, 911

Lin, Y.-T., Mohr, J. J., \& Stanford, S. A. 2003, ApJ, 591, 749

Loewenstein, M. 2001, ApJ, 557, 573

Loewenstein, M., \& Mushotzky, R. F. 1996, ApJ, 466, 695

Luhman, K. L., Stauffer, J. R., Muench, A. A., et al. 2003, ApJ, 593, 1093

Marigo, P., Bressan, A., \& Chiosi, C. 1996, A\&A, 313, 545

Marigo, P., Bressan, A., \& Chiosi, C. 1998, A\&A, 331, 564

Martinelli, A., Matteucci, F., \& Colafrancesco, S. 1998, MNRAS, 298, 42

Martinelli, A., Matteucci, F., \& Colafrancesco, S. 2000, A\&A, 354, 378

Massey, P. 1998, in The Stellar Initial Mass Function, ed. G. Gilmore, \& D. Howell, ASP Conf. Ser., 193, 17

Matteucci, F. 1992, ApJ, 397, 32

Matteucci, F. 1994, A\&A, 288, 57

Matteucci, F. 1997, Fund. Cosmic Phys., 17, 283

Matteucci, F., \& Gibson, B. K. 1995, A\&A, 304, 11

Matteucci, F., \& Vettolani, G. 1988, A\&A, 202, 21

Mitchell, R. J., Culhane, J. L., Davison, P. J. N., \& Ives, J. C. 1976, MNRAS, 175, 29P

Mobasher, B., Colless, M., Carter, et al. 2003, ApJ, 587, 605

Mobasher, B., \& Trentham, N. 1998, MNRAS, 293, 315

Moretti, A. 2001, Evoluzione chimica del mezzo intra-cluster, Master Thesis, Padua University

Mori, M., \& Burkert, A. 2000, ApJ, 538, 559 
Mushotzky, R., Loewenstein, M., Arnaud, K. A., et al. 1996, ApJ, 466, 686

Mushotzky, R. F. 1999, in ed. A. J. Bunker, \& W. J. M. van Breugel, The Hy-Redshift Universe: Galaxy Formation and Evolution at High Redshift, ASP Conf. Ser., 193, 323

Nath, B. B., \& Chiba, M. 1995, ApJ, 454, 604

Padoan, P., \& Nordlund, A. 2002, ApJ, 576, 870

Padoan, P., Nordlund, A., \& Jones, B. J. T. 1997, MNRAS, 288, 145

Pagel, B. E. J. 1997, Nucleosynthesis and chemical evolution of galaxies (Cambridge University Press)

Pei, Y. C., \& Fall, S. M. 1995, ApJ, 454, 69

Pellegrini, S., \& Ciotti, L. 2001, in ed. R. Fusco-Femiano, \& F. Matteucci, Chemical Evolution of Intracluster and Intergalactic Medium, ASP Conf. Ser., 53, 65

Pettini, M. 2000, in The First Stars, ed. A. Weiss, T. Abel, \& V. Hill (Springer), 305, also [astro-ph/0001075]

Pipino, A., Matteucci, F., Borgani, S., \& Biviano, A. 2002, New Astron., 7, 227

Portinari, L., Chiosi, C., \& Bressan, A. 1998, A\&A, 334, 505

Press, W. H., \& Schechter, P. 1974, ApJ, 187, 425

Reiprich, T. H., \& Böhringer, H. 2002, ApJ, 567, 716

Renzini, A. 1997, ApJ, 488, 35

Renzini, A. 2000, in Large Scale Structure in the X-ray Universe, ed. M. Plionis, \& I. Georgantopoulos (Paris, France: Atlantisciences), 103

Renzini, A., \& Ciotti, L. 1993, ApJ, 416, L49

Renzini, A., Ciotti, L., D’Ercole, A., \& Pellegrini, S. 1993, ApJ, 419, 52

Romano, D., Matteucci, F., \& Danese, L. 2002, in Chemical enrichment of intracluster and intergalactic medium, ed. R. Fusco-Femiano, \& F. Matteucci, ASP Conf. Ser., 253, 299

Roussel, H., Sadat, R., \& Blanchard, A. 2000, A\&A, 361, 429

Scalo, J., Vazquez-Semadeni, E., Chappell, D., \& Passot, T. 1998, AJ, 112,948
Schechter, P. 1976, ApJ, 203, 297

Schmidt, M. 1959, ApJ, 129(S), 243

Serlemitsos, P. J., Smith, B. W., Boldt, E. A., Holt, S. S., \& Swank, J. H. 1977, ApJ, 211, L63

Silk, J. 2003, MNRAS, 343, 249

Smith, D. A., Wilson, A. S., Arnaud, K. A., Terashima, Y., \& Young, A. J. 2002, ApJ, 565, 195

Steidel, C. C., Adelberger, K. L., Giavalisco, M., Dickinson, M., \& Pettini, M. 1999, ApJ, 519, 1

Tamura, T., Bleeker, J. A. M., Kaastra, J. S., Ferrigno, C., \& Molendi, S. 2001, A\&A, 379, 107

Tantalo, R., Chiosi, C., Bressan, A., Marigo, P., \& Portinari, L. 1998, A\&A, 335, 823

Thielemann, F. K., Nomoto, K., \& Hashimoto, M. K. 1993, in Origin and evolution of the elements, ed. C. M. N. Prantzos, \& VangioniFlam E. (Cambridge University Press), 297

Thomas, D. 1999, in Chemical Evolution from Zero to High Redshift, ed. J. R. Walsh, \& M. R. Rosa (Springer-Verlag), 197

Tinsley, B. M. 1980, Fund. Cosm. Phys., 5, 287

Tornatore, L., Borgani, S., Springel, V., et al. 2003, MNRAS, 342, 1025

Trentham, N. 1994, Nature, 372, 157

Trentham, N. 1998, MNRAS, 294, 193

Trentham, N., \& Mobasher, B. 1998, MNRAS, 299, 488

Valdarnini, R. 2002, MNRAS, 339, 1117

Vigroux, L. 1977, A\&A, 56, 473

White, D. A. 2000, MNRAS, 312, 663

White, S. D. M., Navarro, J. F., Evrard, A. E., \& Frenk, C. S. 1993, Nature, 366, 429W

White, S. D. M., \& Rees, M. J. 1978, MNRAS, 183, 341

Woosley, S. E., \& Weaver, T. A. 1995, ApJS, 101, 181

Worthey, G., Dorman, B., \& Jones, L. A. 1996, ApJ, 504, 835

Zepf, S. E., \& Silk, J. 1996, ApJ, 466, 114 